

\section{Hypertrophic cardiomyopathy-linked variants of cardiac myosin binding protein C3 display altered molecular properties and actin interaction}

Sahar I. DAAS $\uparrow \dagger$, Khalid FAKHRO $\uparrow \#$, Angelos THANASSOULAS§, Navaneethakrishnan KRISHNAMOORTHY † $^{\wedge}$, Alaaeldin SALEH*, Brian L. CALVER ${ }^{z}$, Bared SAFIEHGARABEDIAN*, Egon TOFT*, George NOUNESIS $\S, F$. Anthony LAI* ${ }^{z_{0}}$ and Michail NOMIKOS*1

\$Translational Medicine, Sidra Medicine, Doha, Qatar

$\uparrow$ College of Health and Life Sciences, Hamad Bin Khalifa University, Doha, Qatar

\#Weill Cornell Medical College, Doha, Qatar

$\S$ National Center for Scientific Research “Demokritos”, Aghia Paraskevi, Greece

${ }^{\wedge}$ Heart Science Centre, National Heart \& Lung Institute, Imperial College London, UK

${ }^{*}$ College of Medicine, Member of QU Health, Qatar University, Doha, Qatar

${ }^{z}$ College of Biomedical \& Life Sciences, Cardiff University, UK

${ }^{\circ}$ Biomedical Research Center, Qatar University Doha, Qatar

${ }^{1}$ Correspondence to:

Michail Nomikos: mixosn@yahoo.com or mnomikos@qu.edu.qa

College of Medicine, Member of QU Health

Qatar University

Doha, PO BOX: 2713, Qatar

Phone: +974 44037846

Abbreviations: HCM, hypertrophic cardiomyopathy; $c-M Y B P C 3$, cardiac myosin binding protein $\mathrm{C} 3$; $\mathrm{CD}$, circular dichroism; $\mathrm{GuHCl}$, guanidine hydrochloride 


\section{ABSTRACT}

The most common inherited cardiac disorder, hypertrophic cardiomyopathy (HCM), is characterized by thickening of heart muscle, for which genetic mutations in cardiac myosinbinding protein $\mathrm{C} 3$ ( $c-M Y B P C 3$ ) gene, is the leading cause. Notably, patients with HCM display a heterogeneous clinical presentation, onset and prognosis. Thus, delineating the molecular mechanisms that explain how disparate $c-M Y B P C 3$ variants lead to HCM is essential for correlating the impact of specific genotypes on clinical severity. Herein, five $c-M Y B P C 3$ missense variants clinically associated with HCM were investigated; namely V1 (R177H), V2 (A216T), V3 (E258K), V4 (E441K) and double mutation V5 (V3+V4), all located within the C1 \& $\mathrm{C} 2$ domains of MyBP-C, a region known to interact with sarcomeric protein, actin. Injection of the variant complementary RNAs in zebrafish embryos were observed to recapitulate phenotypic aspects of HCM in patients. Interestingly, V3- and V5-cRNA injection produced the most severe zebrafish cardiac phenotype, exhibiting increased diastolic/systolic myocardial thickness and significantly reduced heart rate compared to control zebrafish. Molecular analysis of recombinant $\mathrm{C} 0-\mathrm{C} 2$ protein fragments revealed that $c-M Y B P C 3$ variants alter the $\mathrm{C} 0-\mathrm{C} 2$ domain secondary structure, thermodynamic stability and importantly, result in a reduced binding affinity to cardiac actin. V5 (double mutant), displayed the greatest protein instability with concomitant loss of actin binding function. Our study provides specific mechanistic insight into how $c-M Y B P C 3$ pathogenic variants alter both functional and structural characteristics of $\mathrm{C} 0-\mathrm{C} 2$ domains leading to impaired actin interaction and reduced contractility, which may provide a basis for elucidating the disease mechanism in HCM patients with $c$ MYBPC3 mutations.

\section{Keywords:}

Hypertrophic cardiomyopathy, Cardiac myosin binding protein $\mathrm{C} 3, c-M Y B P C 3$ mutations, Zebrafish, 


\section{Introduction}

Hypertrophic cardiomyopathy $(\mathrm{HCM})$ is a common inherited heart disease with an estimated prevalence of $2-5$ per $1000[1,2]$ that is characterized by cardiac impairment linked to increased left ventricular wall thickness, diastolic dysfunction, interstitial fibrosis and myocyte disarray [3, 4]. Clinical presentation of $\mathrm{HCM}$ patients is heterogeneous, ranging from asymptomatic or mildly symptomatic, to distinct features such as dyspnea, angina, syncope, leading in some cases to progressive heart failure $[1,5,6]$. HCM is a genetic autosomal dominant disease characterized by extensive allelic heterogeneity affecting both sarcomeric and sarcomere-related proteins. Variants of the cardiac myosin binding protein $\mathrm{C} 3$ (c-MYBPC3) gene encoding the sarcomeric protein MyBP-C are the most common genetic cause of HCM, representing $40-50 \%$ of all $\mathrm{HCM}$ mutations $[1,7,8]$. Variants with different pathogenicity classification are associated with heterogeneous HCM clinical presentation, disease onset and prognosis [9-13]. Thus, analysis of structural-functional correlations is necessary to understand the molecular mechanisms by which these variants lead to HCM. It has been hypothesized that $c$ MYBPC3 missense mutations directly disrupt the topology of MyBP-C by causing protein misfolding that impairs protein function [14]. The N-terminal domains of MyBP-C - C0, C1 motif and $\mathrm{C} 2$ were previously found to play significant roles in regulating cardiac muscle contraction via their interaction with myosin [15] and actin [16], while its C-terminus was recently reported to directly interact with cardiac ryanodine receptor type 2 (RyR2) [17]. Considering the spectrum of HCM phenotypes described, we hypothesized that missense variation may disparately impact the structure of the protein and differentially affect its functional interactions $[11,18-20]$; hence, in this study we selected five $c-M Y B P C 3$ variants linked with HCM for structural and functional characterization. Three variants (V1-3) (V1, 
Arg177His; V2, Ala216Thr; V3, Glu258Lys) all located within the C1 domain, and V4 (Glu441Lys) within C2 domain were identified in an Egyptian HCM study [21], while V5 is a double mutant (V3 \& V4) that has been associated with a severe, early-onset HCM phenotype [11]. We hypothesized that these variants affect the biophysical and biochemical characteristics of MyBP-C, altering the functional properties of the $\mathrm{C} 1$ and $\mathrm{C} 2$ domains specifically leading to altered MyBP-C-actin association within the sarcomere. To characterize the in vivo effects of these variants, we employed a zebrafish model to elucidate morphometric consequences on cardiac function after synthetic mRNA injections. We also generated the mutant and wild-type N-terminal MyBP-C C0-C2 domains as recombinant proteins to examine their biophysical and biochemical properties, as well as their in vitro interaction with cardiac actin. Injection of zebrafish embryos with synthetic mRNA corresponding to the five MyBP-C variants produced atypical cardiac morphology and impaired cardiac function recapitulating the human HCM phenotype. Collectively, our results provide insight into the molecular impact of variant-specific c-MYBPC3 genotype on MyBP-C biophysical characteristics, protein stability and functional interaction with cardiac actin, contributing to a better understanding of HCM genotype-clinical severity correlation. 


\section{Experimental}

\section{Materials and methods}

\section{Classification of identified variants}

We used the ClinVar database (https://www.ncbi.nlm.nih.gov/clinvar/) for $c-M Y B P C 3$ variant selection with variable pathogenicity classification as: pathogenic, nonpathogenic, or as a variant of unknown clinical significance (unlikely to be pathogenic, uncertain, likely to be pathogenic).

\section{Zebrafish model}

All procedures were performed in conformity with the AAALAC International, Guidance on the housing and care of Zebrafish (Danio rerio) [22]. The study protocol was approved by Qatar University IACUC Office under project approval number QU-IACUC 015/2014. Zebrafish were raised and maintained at $28^{\circ} \mathrm{C}$ on a $14: 10$-h light-dark cycle and fed Artemia nauplii. Wildtype $(A B)$ strain of zebrafish was used for our experiments.

\section{Human $c$-MYBPC3 missense mutation in vitro transcription of mRNA}

Wild-type (WT) $c$-MYBPC3 cDNA (NM 001044349) and the five mutant constructs were cloned into pcDNA-DEST47 vector (Life Technologies) to generate human WT $c$ MYBPC3 mRNA and mRNA encoding the four single missense variants : V1 (c.530G $>$ A), V2 (c.646G $>$ A), V3 (c.772G $>$ A), V4 $($ c.1321G $>$ A) as well as the double variant V5 (c.772G $>$ A + c. $1321 \mathrm{G}>\mathrm{A}$ ) (Figure 1). RNA transcription was performed using the mMESSAGE mMACHINE T7 Transcription Kit, Cat \# AM1344 and Poly(A) Tailing Kit, Cat \# AM1350 (ThermoFisher Scientific). 


\section{Morpholino design and synthetic mRNA injection}

The $c$-mybpc3 morpholino (MO:5'-CTCTGGCATCCTGGTTGAGTGTCCC-3', Gene Tools, USA) targeted against the translational start site was injected into WT strain zebrafish embryos at one cell stage to knock down zebrafish endogenous $c$-mybpc3 [23]. In the mRNA experiments, $c$-mybpc3 MO (2.5 ng) was co-injected with human $c$-MYBPC3 mRNA (100 pg) for the corresponding missense $c-M Y B P C 3$ variants. At least 50 embryos were injected in each group.

\section{Quantitative measurements of zebrafish cardiac size and function}

Injected zebrafish embryos were raised at $28^{\circ} \mathrm{C}$ for 3 days and individually removed from the $28^{\circ} \mathrm{C}$ incubator immediately prior to measurement to minimize the effect of environmental temperature on cardiac function. Zebrafish embryos were mounted in 3\% methylcellulose prior to imaging. The cardiac phenotypes were recorded at 60 frames/second for 3 seconds at $150 \mathrm{X}$ using a stereomicroscope (SteREO Zeiss LUMAR.V12) equipped with USB 2.0 monochrome camera (The Imaging Source).

\section{Measurement of zebrafish ventricular myocardial thickness}

Structural analysis of the zebrafish ventricle was performed by analysis of $2 \mathrm{D}$ images at specific time points. Recorded videos were imported into Image J software (version 1.45s public domain software, http://imagej.nih.gov/ij) and ventricular end-diastole and ventricular endsystole images were extracted. The endocardial and myocardial boundaries were traced to calculate the ventricular wall thickness as the average thickness between these two regions. 


\section{Heart Rate Measurements}

Recorded videos were imported into DanioScope software (Noldus, version 1.0.109) as a compressed AVI file, where a subset of the beating heart was outlined and heart rate was calculated.

\section{Statistical analysis}

Statistical analysis was performed using GraphPad Prism 7. Multiple group comparisons were analyzed by one-way ANOVA. Significant difference between groups was expressed using p values: ${ }^{*} \mathrm{p}<0.05, * * \mathrm{p}<0.01, * * * \mathrm{p}<0.001, * * * * \mathrm{p}<0.0001$

\section{Molecular Modeling}

The complex MyBP-C structure of C1-C2 domains was obtained from our previous molecular dynamics study of $10 \mathrm{~ns}$ simulations at physiological conditions, with all the parameters as described earlier [11]. The representative structures of WT MyBP-C and its variants from the molecular dynamics simulations were selected using cluster analysis. In this, 1000 structures of each variant trajectory were classified into clusters based on their structural deviations. A structure from the top ranked cluster was chosen for representation, as it was a frequently occurring conformation. These simulated structures utilized PyMol (www.pymol.org) for analysis of the secondary structural elements. To quantify the structural components, we used a secondary structure assignment program in PyMOL ("STRIDE") to calculate all the elements. In addition, to map the structural deviations of variants from the WT, we used molecular superposition of the representative structures by aligning using the graphical interface in 
PyMOL. All protein cartoons were generated with high ray trace mode with depth cue to give 3D representation of the molecular models.

\section{Plasmid construction}

Human $c$-MYBPC3 cDNA (NM_001044349) was amplified by PCR from pcDNADEST47, using Phusion polymerase (Finnzymes) and the appropriate primers to incorporate 5'EcoRI and 3'-NotI sites. $c-M Y B P C 3$ was then cloned into pHSIE vector. The primers used for the amplification of $\quad$-MYBPC3 3 'GGAAGGTACCATGGCTGATCAGCTGACCGAAG-3' (forward) and 5'GCAAGCGGCCGCTCATTTTGCAGTCATCATCTGTAC-3’' (reverse).

\section{Prokaryotic expression, purification and intein cleavage of $\mathrm{MyBP}-\mathrm{C} \mathrm{C0}-\mathrm{C} 2$ domain recombinant proteins}

For MyBP-C-fusion protein expression (6xHis-SUMO2-intein-C0-C2), Escherichia coli [BL21-CodonPlus(DE3)-RILP; Stratagene] were transformed with the appropriate plasmid. The presence of the SUMO fusion-tag facilitated enhanced solubility of the recombinant protein in bacterial cells $[24,25]$. Cells were cultured at $37^{\circ} \mathrm{C}$ until the optical density at $600 \mathrm{~nm}$ reached 0.6. Fusion protein expression was then induced for $18 \mathrm{~h}$ at $16^{\circ} \mathrm{C}$ with $0.1 \mathrm{mM}$ IPTG (isopropyl $\beta$-D-thiogalactopyranoside). Bacterial cell pellets were harvested by centrifugation at $6,000 \mathrm{~g}$ for 15 minutes, $4^{\circ} \mathrm{C}$ and then resuspended in $20 \mathrm{ml}$ of buffer A $(50 \mathrm{mM}$ Tris, $\mathrm{pH} 8.5,300 \mathrm{mM} \mathrm{NaCl}$, $25 \mathrm{mM}$ imidazole). Cells were lysed by mild sonication then partitioned into soluble and insoluble fractions by centrifugation at $20,000 \mathrm{~g}$ for 20 minutes at $4^{\circ} \mathrm{C}$ and the supernatant containing the soluble protein fraction was transferred into a fresh tube. Supernatant was then 
applied to a $1 \mathrm{ml} \mathrm{Ni}-\mathrm{NTA}$ column pre-equilibrated in buffer A. To avoid auto-cleavage by intein protease, all the following purification steps were performed at $4^{\circ} \mathrm{C}$ using ice-cold buffers. After binding, the column was washed with $10 \mathrm{x}$ column volumes of buffer A followed by an additional wash (10 x column volumes) of cleaving buffer (PBS buffer supplemented with 40 $\mathrm{mM}$ Bis-Tris and $25 \mathrm{mM}$ imidazole, $\mathrm{pH}$ 6.0). After the second wash, the column was sealed and incubated at room temperature for $3 \mathrm{~h}$ to allow intein cleavage. After intein cleavage, $5 \mathrm{ml}$ of cleaving buffer was used to elute the cleaved $\mathrm{C} 0-\mathrm{C} 2$ (MyBP-C) proteins from the resin. The remaining proteins bound to the column were eluted with $10 \mathrm{ml}$ of cleaving buffer supplemented with $500 \mathrm{mM}$ imidazole, and the eluted fractions were collected and analysed by $12 \%$ SDSPAGE electrophoresis.

\section{Western Blotting}

Purified recombinant MyBP-C $\mathrm{C} 0-\mathrm{C} 2$ proteins were analyzed by SDS-PAGE electrophoresis with Coomassie Brilliant Blue staining and immunoblot analysis performed as described previously [26]. Proteins were probed with a polyclonal anti-MyBP-C antibody (1:1,000 dilution) (Abgent, Cat \# AP12436a) and immunoreactive proteins were detected by ECL Chemiluminescence Detection Kit (Thermofisher Scientific, Cat \#32106).

\section{Circular Dichroism (CD) Analysis}

CD measurements were conducted using a JASCO-715 spectropolarimeter with a Peltier type cell holder. Wavelength scans in the $190 \mathrm{~nm}$ to $260 \mathrm{~nm}$ range (far-UV region) were performed in Quartz SUPRASIL (HELLMA) precision cells of $0.1 \mathrm{~cm}$ path length, using a wavelength step of $0.2 \mathrm{~nm}$, a scan rate of $20 \mathrm{~nm} \mathrm{~min}^{-1}$, response time $1 \mathrm{~s}$ and $1 \mathrm{~nm}$ bandwidth. 
Buffer spectra were collected and subtracted from all scans. The concentration of all protein samples was $0.2 \mathrm{mg} / \mathrm{ml}$.

Thermal denaturation data were collected by heating the protein sample from $20^{\circ}$ to $90^{\circ} \mathrm{C}$ using a scan rate of $1.5 \mathrm{~K} / \mathrm{min}$ and monitoring signal changes at $208 \mathrm{~nm}$. Spectral parameters for these measurements were: $1 \mathrm{~s}$ step resolution, $0.5 \mathrm{~s}$ response time, sensitivity $25 \mathrm{mdeg}$ and bandwidth $1 \mathrm{~nm}$. Data analysis software (CDNN version 2.1) was used to deconvolute the collected far-UV CD spectra in order to determine the protein's $\alpha$-helix, anti and parallel $\beta$-sheet, $\beta$-turn and random coil content.

\section{Chemical Denaturation}

Fluorescence spectra were collected on a PTI QuantaMaster 400 steady-state fluorescence system. Tryptophan was selectively excited at $295 \mathrm{~nm}$ and emission spectra were collected between 310 to $450 \mathrm{~nm}$ using a $4 \mathrm{ml}$ quartz cuvette (Hellma) with a path length of 10 $\mathrm{mm}$. Scan rate was set to $100 \mathrm{~nm} \min ^{-1}$ with a response time of $1 \mathrm{sec}$. Slit width for both emission and excitation were $2 \mathrm{~nm}$. The protein species concentration used for all fluorescence experiments was $0.2 \mathrm{mg} / \mathrm{ml}$. All fluorescence spectra were corrected for background intensity as measured with pure buffer. In a typical experiment, small amounts of an $8 \mathrm{M}$ guanidine hydrochloride solution were gradually added in the cuvette along with the appropriate amount of concentrated protein solution in order to keep the MyBP-C concentration in the cell constant. The mixture was stirred using a small magnetic stir bar for $1 \mathrm{~min}$ and incubated in the dark for $15 \mathrm{~min}$, at which point the fluorescence emission spectrum of the sample was collected. The titration was carried on until a $5 \mathrm{M}$ final concentration of guanidine hydrochloride was reached 
inside the cuvette. Mock titrations with the addition of buffer instead of guanidine hydrochloride were collected and the corresponding spectra were subtracted from the original data.

The final data were fitted using the following equation [27]:

$$
F=\frac{\left(a_{N}+b_{N} \cdot[D]\right)+\left(a_{D}+b_{D} \cdot[D]\right) \cdot e^{-\left(\frac{\Delta G}{R T}\right)}}{1+e^{-\left(\frac{\Delta G}{R T}\right)}}
$$

where: $\Delta G=m\left(\left[D^{50 \%}\right]-[D]\right),[D]$ is the concentration of the denaturant in the cuvette, $\left[D^{50 \%}\right]$ is the concentration of denaturant at the midpoint of the transition, $\mathrm{m}$ is the slope of the denaturation transition, $\mathrm{R}$ is the universal gas constant, $\mathrm{T}$ is the experimental temperature, $\mathrm{a}_{\mathrm{N}}$ and $b_{N}$ the intercept and slope of the native state baseline, while $a_{D}$ and $b_{D}$ are the corresponding values of the denatured state baseline.

\section{Co-sedimentation Cardiac Actin Binding Assays}

Co-sedimentation assays were performed as previously described [28]. Actin Binding Protein Biochem Kit (Cytoskeleton, Cat \# BK001) and bovine cardiac actin (Cytoskeleton, Cat \# AD99-B) were used. Bovine cardiac actin was prepared in General Actin Buffer (5 mM Tris-HCl $\mathrm{pH}$ 8.0, 0.2 mM CaCl2) (Cytoskeleton, Cat \# BSA01) and supplemented with 0.2 mM ATP and $0.5 \mathrm{mM}$ DTT prior to use. Purified N-terminal C0-C2 MyBP-C protein fragments were dialyzed using Vivaspin tubes (Sartorius, Vivaspin 20). Initially, optimization experiments were performed using $2 \mu \mathrm{M}$ of wild type MyBP-C C0-C2 and 1, 10, 20 or $30 \mu \mathrm{M}$ c-actin, in order to identify the optimal concentration in which saturated binding between MyBP-C and c-actin is achieved. After the optimal concentrations were determined, $30 \mu \mathrm{M}$ of c-actin was incubated with $2 \mu \mathrm{M}$ of each MyBP-C C0-C2 recombinant protein (wild-type and mutants) for $30 \mathrm{~min}$ at room temperature. For our co-sedimentation experiments, incubation of c-actin with $\alpha$-actinin 
protein served as our positive control, while incubation of c-actin with BSA served as our negative control. After incubation, samples were centrifuged at $14,000 \mathrm{~g}$ for 1 hour at $24{ }^{\circ} \mathrm{C}$. Supernatants were carefully removed, mixed with $10 \mu 1$ of 5 x Laemmli reducing-sample buffer and analyzed by SDS-PAGE electrophoresis and Coomassie brilliant blue staining. Finally, to estimate the relative binding of c-actin to the various recombinant proteins, band intensities appearing in the stained gels were measured by densitometric analysis and the data analyzed using GraphPad Prism 7.

\section{Results}

\section{$c-M Y B P C 3$ variants are associated with distinct HCM phenotype}

The elucidation of genotype-phenotype associations provides a crucial parameter to help predict the impact of a specific variant on HCM clinical severity. To investigate the mechanism by which different $c$-MYBPC3 variants lead to cardiac impairment, we studied four single missense mutations: V1 (c.530G >A, p.R177H), V2 (c.646G>A, p.A216T ), V3 (c.772G>A, p.E258K), V4 (c.1321G>A, p.E441K) in addition to a double mutation V5 (V3+V4), (Figure 1). HCM patients expressing these variants exhibited a range of clinical symptoms and variable degrees of ventricular hypertrophy as determined by septum and wall thickness (Table 1). Multiple in silico algorithms, and/or population frequency databases defined the five variants' pathogenicity classification that varied from uncertain significance to certainly pathogenic. Furthermore, the patient carrying the double mutation (V5) had an early-onset disease at age 21 and died at the age of 42. Conversely, patients carrying single mutations (V1, V2, V3, and V4) had lower severity symptoms leading to heart failure later on in life (Table 1) suggesting that 
patients expressing different $c-M Y B P C 3$ variants can present with typical HCM clinical heterogeneity in regard to disease severity, onset and prognosis.

\section{Modeling HCM patients' c-MYBPC3 variants using zebrafish}

Zebrafish (Danio rerio) is a powerful vertebrate animal model to study cardiac function due to its embryonic transparency [29]. To assess variants' genotype-phenotype associations, we injected synthetic mRNA encoding $c$-MYBPC3 variants at the zebrafish one-cell stage and examined their cardiac morphology and function. The injection of synthetic mRNA resulted in aberrant cardiac morphology with altered ventricular sizes and function when examined at 3 days post-fertilization (dpf) (Figure 2, supplemental video 1). V1, V2 and V4 displayed notably different ventricular morphology to the control group. However, V5 and V3 exhibited a greater disparity in ventricular phenotype compared to other variants (Figure 2B, supplemental video 1). These results demonstrate that $c-M Y B P C 3 \mathrm{~V} 5$ and V3 zebrafish cardiac phenotypes correlate with their pathogenicity classification in HCM patients, as both are predicted to be pathogenic class 5 (Table 1). Thus, expression of these variants in the zebrafish model essentially recapitulated the HCM pathological cardiac phenotype in comparison to controls (Figure 2A-B).

\section{Zebrafish $c$-MYBPC3 models develop increased ventricular myocardial thickness}

Cardiac morphometric measurement of the ventricular myocardial wall thickness of $c$ MYBPC3 variant-injected embryos was determined at both diastole and systole (Figure 2C-D). All $c-M Y B P C 3$ variants resulted in a significantly increased ventricular myocardial thicknesses 
in comparison to control group. The V5 (V3 \& V4) double mutation, and V3 (E258K) exhibited the most severe myocardial hypertrophic phenotype with increased diastolic / systolic myocardial thickness at $15.6 / 14.6 \mu \mathrm{m}(\mathrm{p}<0.0001)$ and $14.2 / 12.9 \mu \mathrm{m}(\mathrm{p}<0.0001)$, respectively. While thickness for V1 (R177H) was $12.7 / 12.5 \mu \mathrm{m}(\mathrm{p}<0.0001)$, V2 (A216T) yielded $11.5 / 11.7$ $\mu \mathrm{m}(\mathrm{p}<0.001)$ and V4 $(\mathrm{E} 441 \mathrm{~K})$ displayed $12.1 / 12.3 \mu \mathrm{m}(\mathrm{p}<0.0001)$ in contrast to control zebrafish at $10.1 / 9.5 \mu \mathrm{m}$ (Figure 2C-D). Zebrafish cardiac phenotype resembled the human ventricular hypertrophic phenotype as HCM patients exhibit increased myocardial wall thickness with a heterogeneous clinical presentation among patients [30].

\section{Zebrafish $c$-MYBPC3 models develop impaired cardiac contractility}

To examine whether the $c-M Y B P C 3$ variants elicit different effects on cardiac function, we assessed the heart rate of injected zebrafish as an indicator of cardiac contractile impairment. Introduction of both V3 and V5 synthetic mRNA significantly affected the average heart rate resulting in a $23 \%$ reduction for both groups; 113 beats per minute (bpm) compared to the level observed in zebrafish controls at $146 \mathrm{bpm}$. In contrast, there was a moderate decrease $(<10 \%)$ for V1, V2, and V4, at 138, 143 and 133 bpm, respectively; however, these still represent cardiac contractile impairment (Figure 2E). These findings suggest that genotype specificity contributes to HCM phenotypic severity as the V5 double mutation produces early-onset and progression to end-stage HCM (Table 1). Indeed, mRNA injection corresponding to V5 variant in zebrafish resulted in the most severe heart rhythm impairment (Figure 2B-E).

\section{Bioinformatics analysis of $c-M Y B P C 3$ variant effect on functional domains}


The severe phenotypes of V3 and V5 led us to study structure-function relationships at protein level, which may reveal molecular determinants that underlie genotype to phenotypic expressions. Several studies have reported that the region C1-C2 of MyBP-C plays a central role in the interactions with actin and myosin proteins within the sarcomere [31, 32]. Our molecular model of the $\mathrm{C} 1-\mathrm{C} 2$ region of the WT protein suggests this linker segment (MyBP-C motif) may hold the key sites for protein phosphorylation (Figure 3A). The secondary structural elements of the domains $\mathrm{C} 1$ and $\mathrm{C} 2$ in the WT comprises $\beta$-sheets, however, the linker/motif is highly $\alpha$ helical in nature. Both the V3 and V5 molecular models deviated structurally and were arranged differently from WT, with the majority of these rearrangements observed in the motif region (Figure 3B-C). The variants induced changes in the secondary structural elements of the $\mathrm{C} 1$ and $\mathrm{C} 2$ domains and in the intervening motif region, where the impact was in particular, higher in V5 (Figure 3C) in comparison to V3. Interestingly, V3 appeared to be flexible, while V5 appeared to be rigid compared to WT in the RMSD (root mean square deviation) calculation of the representative structures from molecular dynamics simulations (Figure 3D). The secondary structural changes shown in the graph (Figure 3E) provides an overview of the intra-molecular consequences that induce structural rearrangements in both V5 and V3. Here, the V3 model presents reduced $\beta$-sheets and increased coils compared to WT, whereas V5 showed inverse behavior. This correlates with our deviation analysis and reflects V3 flexibility with increase of coils, and the rigid V5 upon reduction of coils with increased $\beta$-sheets. Overall, this protein modeling helps to provide a molecular insight into the structural consequences of MyBP-C variants.

\section{Circular dichroism (CD) spectroscopy and thermal stability analysis of MyBP-C variants}


To investigate the impact of these $c-M Y B P C 3$ variants on the biophysical and biochemical properties of WT MyBP-C protein, we generated prokaryotic expression plasmid constructs encoding the $\mathrm{C} 0-\mathrm{C} 2 \mathrm{~N}$-terminal domains of WT and the five variants (Figure 4A). The purified recombinant $\mathrm{C} 0-\mathrm{C} 2$ fragment proteins were highly soluble and migrated on SDS-PAGE according to their predicted molecular weight of $\sim 50 \mathrm{kDa}$, as shown in both Coomassie stained gel and western blot analysis using MyBP-C N-terminal antibody (Figure 4B). To better understand the structural effects of these $c-M Y B P C 3$ variants, we measured far-UV CD spectra (Figure 4C) and we determined the secondary structure elements (Table 2) of all protein samples at $25^{\circ} \mathrm{C}$. It was evident that all variants disrupted the native structure of the $\mathrm{C} 0-\mathrm{C} 2$ proteins, leading to conformations with significantly different secondary structure characteristics when compared to WT (Table 2). At elevated temperatures $\left(\sim 90^{\circ} \mathrm{C}\right)$, all protein species retain most of their secondary structure characteristics (Supplemental material, Tables 1-6). However, for WT, V1 and V2, we observed an increase in random coil content with a concurrent loss of helicity, reflecting partial unfolding of the structure. At the same time, the $\beta$-sheet content of the protein fragments remained relatively intact, revealing a central core of $\beta$-sheets with high thermal stability. For V3, V4 and V5 only small changes in the secondary structure content were observed. This was also evident from the relatively small molar ellipticity change recorded in the far-UV spectra throughout the $20^{\circ} \mathrm{C}-90^{\circ} \mathrm{C}$ temperature range. Most likely, the observed changes involved spatial rearrangement of the secondary structure elements without significant unfolding. CD melting curves were collected to analyze the thermal denaturation profiles at $208 \mathrm{~nm}$ over the temperature range $20^{\circ} \mathrm{C}-90^{\circ} \mathrm{C}$ (Figure 5). The relative signal change for $\mathrm{V} 3$, V4 and V5 was small, resulting in curves with lower signal to noise ratio than the corresponding profiles of WT, $\mathrm{V} 1$, and V2. The thermal denaturation curve of WT, V1, V2, V3 and V4 revealed the presence of 
two consecutive unfolding transitions; the first thermal transition occurs in the temperature range $50^{\circ} \mathrm{C}-65^{\circ} \mathrm{C}$ with the second at $65^{\circ} \mathrm{C}-90^{\circ} \mathrm{C}$. Interestingly, in contrast to other variants, the melting profile of V5 exhibits only the unfolding transition at $50^{\circ} \mathrm{C}-65^{\circ} \mathrm{C}$, suggesting that no further protein aggregation occurred after $\sim 60^{\circ} \mathrm{C}$ for this fragment. A possible mechanism for this complex thermal behavior involves the formation of oligomers from monomer protein fragments (first transition). As the temperature of the sample increases, partial unfolding and structural rearrangements of the proteins, dictated by further changes in their aggregation state, result in a second thermal transition. A similar thermal behavior has been previously reported for other MyBP-C motifs $[33,34]$ with dynamic light scattering studies confirming the formation of aggregates upon heating [34].

\section{Chemical denaturation profiles for the WT and mutant MyBP-C proteins}

To determine the thermodynamic stability of mutant MyBP-C protein fragments, we tested the reversibility of chemically-induced transitions to calculate the Gibbs free energy change $(\Delta G)$ of the unfolding process (Table 3). Chemical denaturation profiles of WT and MyBP-C recombinant fragment proteins exhibited transitions that were completely reversible and under thermodynamic control, with all samples recovering their initial spectra after dialysis into their original buffer (Figure 6). Interestingly, except for V5, chemical denaturation of all variants lead to conformations with increased stability compared to the WT MyBP-C fragment. Notably, V3 and V4 showed a significant increase in $\Delta \mathrm{G}(\sim 1.5 \mathrm{kcal} / \mathrm{mol})$, while for V1 and V2 this change was less than $0.6 \mathrm{kcal} / \mathrm{mol}$, slightly above the statistical error for these measurements. The recorded V5 destabilizing effect is small and lower than the corresponding statistical error, indicating that V5 has practically no impact on the thermodynamic stability of 
MyBP-C. Thermodynamic analysis for the chemical-induced denaturation of all fragments (Table 3) showed $\Delta \mathrm{G}$ values lower than the typical 5-15 kcal/mol found for most proteins [27].

\section{MyBP-C variants affect cardiac function through impaired actin binding}

MyBP-C is a multi-domain protein with vital structural and regulatory roles. The selected variants are located within the $\mathrm{N}$-terminal region, which encompasses the $\mathrm{C} 1-\mathrm{C} 2$ domains of MyBP-C that is involved in regulating cardiac muscle contraction through interaction with actin within the sarcomere (Figure 4A). The recombinant $\mathrm{C} 0-\mathrm{C} 2$ domains have been previously shown to interact with actin [16]. Thus, we hypothesized that impaired cardiac function phenotypes may be correlated with a reduced ability of MyBP-C to interact with actin. To investigate the specific effects of the variant's structural alterations on actin binding properties, we employed a cosedimentation assay. In this assay, c-actin can be co-sedimented due to its filamentous nature, whereas non-filamentous or non-interacting proteins remain in the supernatant. In the absence of c-actin, WT MyBP-C C0-C2 recombinant protein remained in the supernatant fraction. However, when cardiac actin was added to the assay, WT MyBP-C C0-C2 recombinant protein specifically bound and co-sedimented with c-actin as the recombinant protein was detected in the pellet. After a series of optimization experiments, we found that $2 \mu \mathrm{M}$ of MyBP-C recombinant $\mathrm{C} 0-\mathrm{C} 2$ domains can specifically bind to $30 \mu \mathrm{M}$ actin in a fully saturated manner (Figure 7A). $2 \mu \mathrm{M}$ of each MyBP-C C0-C2 recombinant protein (WT and mutants) was incubated with c-actin at RT for $30 \mathrm{~min}$ and centrifuged for 1 hour at $14,000 \mathrm{~g}$. The experiment was repeated with a different batch of recombinant proteins. The amounts of MyBP-C C0-C2 fragments in the supernatant fractions (unbound fractions) were analyzed by SDS-PAGE and quantified by Coomassie brilliant blue (Figure 7B) and densitometric analysis (Figure 7C). Following densitometric 
analysis and \% normalization of the binding of MyBP-C C0-C2 recombinant proteins to c-actin (Figure 7C), we found that except for V1, all the other variants (V2, V3, V4 and V5) displayed significantly reduced c-actin binding, with V4 and especially V5 showing the most dramatic reductions ( $\sim 38$ and $52 \%$ reduction of their c-actin relative binding affinities, respectively).

\section{Discussion}

HCM is a genetically heterogeneous autosomal dominant cardiac disease [4, 35], with many $c-M Y B P C 3$ variants previously reported in $\mathrm{HCM}$ patients $[1,8]$. Since HCM clinical presentation in patients is also heterogeneous, it is hypothesized that severity is correlated to underlying genetic mutations. Previous studies demonstrated that patients carrying specific mutations have been associated with earlier disease presentation, poorer prognosis, severe disease phenotype, higher prevalence of a family history and sudden cardiac death $[32,36,37]$. Furthermore, most of the childhood-onset disease mutations are missense $(\sim 76 \%)$, while $\sim 60 \%$ of previously reported mutations in adults encode for MyBP-C truncations. This observation suggests that $c-M Y B P C 3$ missense mutations causing HCM may be more severe than truncating mutations, explaining the earlier age of onset [38]. Some studies have established potential correlations in interpreting the phenotypic effect of these variants. However, the exact mechanism by which these variants affect MyBP-C physiological functions within the sarcomere leading to the different clinical phenotypes still remains unclear.

Our current study presents a new model of an in vitro functional genomics approach to investigate the effects of specific genotypes on MyBP-C biophysical and biochemical 
characteristics that may explain HCM clinical severity. We selected five $c-M Y B P C 3$ variants (Figure 1) that represented HCM heterogeneity with different pathogenicity classifications, as V3 and V5 (double mutant) were classified as the most pathogenic variants [11, 18-20]. Importantly, the double mutant V5 and the single mutant V3 exhibited the most severe myocardial hypertrophic phenotype (Table 1). Molecular modeling suggested critical structural consequences for these two variants (Figure 3). Furthermore, our biophysical analysis revealed that all variants lead to distinct protein conformations affecting the secondary structure content (Figure 4) and the thermodynamic stability (Figure 5) of the MyBP-C C0-C2 recombinant proteins. Consequently, distinct alterations of the MyBP-C C0-C2 fragments binding affinity to cardiac actin were observed when we investigated the protein interaction of these variants to cardiac actin (Figure 7).

Using different techniques of the zebrafish 'toolbox', we were able to recapitulate the different HCM phenotypes in the zebrafish model. All $c-M Y B P C 3$ variants resulted in aberrant zebrafish cardiac morphology with altered ventricular size and function (Figure 2, supplemental material, 1). Our zebrafish experiments explored allele pathogenicity and captured specific cardiac phenotypes as effects that are variant-specific. The resultant zebrafish cardiac phenotype accurately resembled the human ventricular hypertrophic phenotype given that HCM patients exhibit increased myocardial wall thickness. Notably, V5 (E441K \& E258K) and V3 (E258K) resulted in the most dramatic zebrafish cardiac impairment presenting with increased ventricular dimension in comparison to other variants. The V5 and V3 diastolic/systolic myocardial thickness was significantly increased and both variants exhibited significant contractile dysfunction with decreased heart rate in contrast to the other variants and wild-type MyBP-C. These results support the established class 5 pathogenicity of these two variants (Table 1). 
Collectively, our zebrafish functional validation suggests an association of genotype to a disease phenotype; and confirmed the in-silico tools of pathogenicity classification of a specific genetic variant. Our findings support previous studies of other MyBP-C variants associated with a severe clinical phenotype [39]. Previous studies have demonstrated that each individual $c$-MYBPC3 patient's phenotype varies with the position of the mutation and its cognate functional domain. It has been suggested that possessing multiple disease-causing sarcomere mutations may be associated with a greater risk for severe disease progression, including sudden cardiac death. This can be interpreted as double (or compound) variants exerting a gene dosage effect in HCM patients [9, 37, 40]. Recent reports using molecular modeling together with clinical and expression data revealed that the missense mutations in the $\mathrm{C} 1-\mathrm{C} 2$ of MyBP-C could be responsible for the severe symptoms studied in selected HCM patients [11, 13, 32]. Accordingly, our study particularly highlights the variants-induced secondary structural modifications in detail and reveal conformational rearrangements of the variants V3 and V5 (enhanced impact), which lead to severe effects on the motif region. As reported previously by many studies $[31,41]$, this motif is a key location for protein phosphorylation and for interaction with actin and/or myosin which serve to regulate the mechanism of muscular contractions. Therefore, the observed data from our modeling and CD-spectroscopy collectively suggest variation-induced secondary structural changes could be detrimental to function, explaining the abnormal phenotypes that we observed in our diseased models of zebrafish. Earlier molecular modeling suggested that V1 and V2 might increase the intra-molecular rigidity due to induced structural changes, resulting in minimal effect on the surface electrostatic properties that are key for binding with actin [13]. Contrastingly, V3 was predicted to significantly alter the structural properties leading to major change in $\mathrm{C} 1$ surface and malfunction of the protein [42]. Significantly, modeling of V5 
conformational changes of $\mathrm{C} 1-\mathrm{C} 2$ domains predicted alterations of the binding of these domains to other MyBP-C domains and other sarcomeric proteins (actin and myosin), in particular by affecting the nearby motif region. These effects possibly explain the early onset and the severity of the observed HCM phenotype [11].

By using the zebrafish model to study the effects of these MyBP-C variants, our data indicate correlation of phenotypic variations to specific molecular mechanisms of altered protein characteristics and binding interactions with c-actin. The recombinant N-terminal, $\mathrm{C} 0-\mathrm{C} 2$ fragment, of V5 and V3 confirmed the model of altered secondary structural elements of the protein. As a result, V5 had only one unfolding transition step in the thermal denaturation curve compared to the other variants, which demonstrated two consecutive unfolding transitions. These observations highlight the fact that these missense variants result in altered protein conformations, which could potentially affect MyBP-C physiological functions within the sarcomere. MyBP-C is a multi-modular structural protein of 11 domains regulating sarcomere organization and contractility $[1,15,43,44]$. It was previously shown that the N-terminal complex, C1-C2 of MyBP-C, regulates cardiac muscle contraction through actin interaction within the sarcomere $[15,28,45,46]$. Our in vitro MyBP-C/cardiac actin co-sedimentation assays confirmed the negative effect of V2, V3, V4 and V5 mutations on the intermolecular interactions of MyBP-C with c-actin and more importantly highlighted the severe effect of the double mutation, V5. Accordingly, V5 relative binding affinity to c- actin was reduced almost $52 \%$ when compared to that of WT. In contrast, V1 variant did not exhibit any significant reduction on its relative binding affinity to c-actin suggesting alternative disease mechanisms rather than altered c-actin binding affinity. As recent studies have shown a significant overlap between the N-terminal of MyBP-C and actin as well as myosin S2 binding sites [47], further 
analysis should be considered to study the impact of these variants on their interaction with myosin. Interestingly, despite the fact that we detected a significant reduction of V3 relative binding affinity to c-actin $(\sim 27 \%)$, earlier studies using the yeast two-hybrid system indicated that MyBP-C N-terminal E258K variant (V3) had reduced affinity to myosin heavy chain subfragment 2, providing a potential alternative mechanism underlying the HCM disease phenotype [48]. Our findings might begin to explain the contractile dysfunction resulting from the different $c-M Y B P C 3$ variants as a result of their divergent biochemical and biophysical characteristics. Interestingly, the effect of the five mutations on cardiac phenotype in our experimental zebrafish model is consistent with previously reports on the patients' clinical severity.

\section{Conclusion}

Although MyBP-C protein has been extensively studied, the exact molecular mechanism(s) by which MyBP-C missense variants lead to severe cardiac disease remain elusive. This is largely due to the lack of a comprehensive understanding of the impact of these mutations on the protein molecular characteristics, as well as on its interactions with other sarcomeric components.

Our current study has clearly demonstrated that the zebrafish model is a very useful in vivo 'tool' to prognose and characterize the pathogenicity of MyBP-C missense mutations identified in patients with cardiac disease. It also provides further evidence for the important role of MyBP-C N-terminal C0-C2 domain on the vital interaction of this protein with actin within the sarcomere. However, taking into consideration that our observations are based on the in vitro biochemical and biophysical characterization of the isolated $\mathrm{C} 0-\mathrm{C} 2$ domains of MyBP-C protein and not of the full-length molecule, further in vivo studies are required to confirm and expand on our findings. We cannot exclude the possibility that the behavior of the full-length wild type 
MyBP-C protein and its corresponding variants might be very different in vivo, necessitating further in vivo functional analysis, which will shed more light into the molecular mechanism(s) that mutations in $c-M Y B P C 3$ gene lead to severe cardiac disease.

\section{ACKNOWLEDGEMENTS}

We thank Ms Rola Salem for her technical assistance with the protein expression and purification experiments.

DECLARATIONS OF INTEREST: The authors declare no financial interests.

\section{FUNDING}

M.N. was supported by internal CMED (QU) funding. A.T. was supported by the IKY scholarships programme through the action entitled "Reinforcement of Postdoctoral Researchers", in the framework of the "Human Resources Development Program, Education and Lifelong Learning” of the National Strategic Reference Framework (NSRF) 2014-2020.

\section{AUTHOR CONTRIBUTIONS}

S.I.D., K.F. and M.N. devised the project strategy. S.I.D., K.F., B.S.G., E.T., G.N., F.A.L. and M.N. designed/analyzed the experiments, which were performed by S.I.D, A.T., A.S., B.L.C., and M.N. Molecular modeling was performed by N.K. Finally, S.I.D. and M.N. prepared the first manuscript draft, which was revised and approved by all authors.

\section{References}

1 Mohamed, I. A., Krishnamoorthy, N. T., Nasrallah, G. K. and Da'as, S. I. (2017) The Role of Cardiac Myosin Binding Protein C3 in Hypertrophic Cardiomyopathy-Progress and Novel Therapeutic Opportunities. J. Cell. Physiol. 232, 1650-1659

2 Semsarian, C., Ingles, J., Maron, M. S. and Maron, B. J. (2015) New perspectives on the prevalence of hypertrophic cardiomyopathy. J. Am. Coll. Cardiol. 65, 1249-1254 
3 Varnava, A. M., Elliott, P. M., Mahon, N., Davies, M. J. and McKenna, W. J. (2001) Relation between myocyte disarray and outcome in hypertrophic cardiomyopathy. Am. J. Cardiol. 88, 275-279

4 Marsiglia, J. D. C. and Pereira, A. C. (2014) Hypertrophic cardiomyopathy: how do mutations lead to disease? Arq. Bras. Cardiol. 102, 295-304

5 Houston, B. A. and Stevens, G. R. (2015) Hypertrophic cardiomyopathy: a review. Clin. Med. Insights Cardiol. 8, 53-65

6 Sen-Chowdhry, S., Jacoby, D., Moon, J. C. and McKenna, W. J. (2016) Update on hypertrophic cardiomyopathy and a guide to the guidelines. Nat. Rev. Cardiol. 13, 651-675

7 Olivotto, I., d'Amati, G., Basso, C., Van Rossum, A., Patten, M., Emdin, M., Pinto, Y., Tomberli, B., Camici, P. G. and Michels, M. (2015) Defining phenotypes and disease progression in sarcomeric cardiomyopathies: contemporary role of clinical investigations. Cardiovasc. Res. 105, 409-423

8 Carrier, L., Mearini, G., Stathopoulou, K. and Cuello, F. (2015) Cardiac myosin-binding protein C (MYBPC3) in cardiac pathophysiology. Gene 573, 188-197

9 Maron, B. J., Maron, M. S. and Semsarian, C. (2012) Double or compound sarcomere mutations in hypertrophic cardiomyopathy: a potential link to sudden death in the absence of conventional risk factors. Heart Rhythm 9, 57-63

10 Fourey, D., Almansoori, G., Care, M., Rakowski, H. and Chan, R. H. (2015) Double or Compound Sarcomere Mutations in Hypertrophic Cardiomyopathy is Not Associated With Worsened Outcomes: A Single Center Cohort Study. Circulation 132, 18559

11 Gajendrarao, P., Krishnamoorthy, N., Selvaraj, S., Girolami, F., Cecchi, F., Olivotto, I. and Yacoub, M. (2015) An investigation of the molecular mechanism of double cMyBP-C mutation in a patient with end-stage hypertrophic cardiomyopathy. J. Cardiovasc. Transl. Res. 8, 232-243

12 Liu, X., Jiang, T., Piao, C., Li, X., Guo, J., Zheng, S., Zhang, X., Cai, T. and Du, J. (2015) Screening mutations of MYBPC3 in 114 unrelated patients with hypertrophic cardiomyopathy by targeted capture and next-generation sequencing. Sci. Rep. 5, 11411

13 Gajendrarao, P., Krishnamoorthy, N., Kassem, H. S., Moharem-Elgamal, S., Cecchi, F., Olivotto, I. and Yacoub, M. H. (2013) Molecular modeling of disease causing mutations in domain C1 of cMyBP-C. PloS one 8, e59206

14 Schlossarek, S., Englmann, D. R., Sultan, K. R., Sauer, M., Eschenhagen, T. and Carrier, L. (2012) Defective proteolytic systems in Mybpc3-targeted mice with cardiac hypertrophy. Basic Res. Cardiol. 107,235

15 Sadayappan, S. and de Tombe, P. P. (2012) Cardiac myosin binding protein-C: redefining its structure and function. Biophys. Rev. 4, 93-106

16 Rybakova, I. N., Greaser, M. L. and Moss, R. L. (2011) Myosin binding protein c interaction with actin characterization and mapping of the binding site. J. Biol. Chem. 286, 2008-2016

17 Stanczyk, P. J., Seidel, M., White, J., Viero, C., George, C. H., Zissimopoulos, S. and Lai, F. A. (2018) Association of cardiac myosin-binding protein-C with the ryanodine receptor channel - putative retrograde regulation? J. Cell Sci. 131, 210443

18 Jaafar, N., Gómez, J., Kammoun, I., Zairi, I., Ben Amara, W., Kachboura, S., Kraiem, S., Hammami, M., Iglesias, S., Alonso, B. and Coto, E. (2016) Spectrum of Mutations in Hypertrophic Cardiomyopathy Genes Among Tunisian Patients. Genet. Test Mol. Biomarkers 20, 674-679

19 Rodríguez-García, M. I., Monserrat, L., Ortiz, M., Fernández, X., Cazón, L., Núñez, L., BarrialesVilla, R., Maneiro, E., Veira, E. and Castro-Beiras, A. (2010) Screening mutations in myosin binding protein C3 gene in a cohort of patients with Hypertrophic Cardiomyopathy. BMC Med. Genet. 11, 67

20 Marsiglia, J. D. C., Batitucci, M. d. C. P., Paula, F. d., Barbirato, C., Arteaga, E. and Araújo, A. Q. d. (2010) Study of mutations causing hypertrophic cardiomyopathy in a group of patients from Espirito Santo, Brazil. Arq. Bras. Cardiol. 94, 10-17 
21 Kassem, H. S., Azer, R. S., Ayad, M. S., Moharem-Elgamal, S., Magdy, G., Elguindy, A., Cecchi, F., Olivotto, I. and Yacoub, M. H. (2013) Early results of sarcomeric gene screening from the Egyptian National BA-HCM Program. J. Cardiovasc. Transl. Res. 6, 65-80

22 Reed, B. and Jennings, M. (2011) Guidance on the housing and care of zebrafish Danio rerio. Southwater: Royal Society for the Prevention of Cruelty to Animals

23 Chen, Y.-H., Pai, C.-W., Huang, S.-W., Chang, S.-N., Lin, L.-Y., Chiang, F.-T., Lin, J.-L., Hwang, J.-J. and Tsai, C.-T. (2013) Inactivation of Myosin binding protein C homolog in zebrafish as a model for human cardiac hypertrophy and diastolic dysfunction. J. Am. Heart Assoc. 2, e000231

24 Nomikos, M., Thanassoulas, A., Beck, K., Vassilakopoulou, V., Hu, H., Calver, B. L., Theodoridou, M., Kashir, J., Blayney, L., Livaniou, E., Rizkallah, P., Nounesis, G. and Lai, F. A. (2014) Altered RyR2 regulation by the calmodulin $\mathrm{F} 90 \mathrm{~L}$ mutation associated with idiopathic ventricular fibrillation and early sudden cardiac death. FEBS Lett. 588, 2898-2902

25 Vassilakopoulou, V., Calver, B. L., Thanassoulas, A., Beck, K., Hu, H., Buntwal, L., Smith, A., Theodoridou, M., Kashir, J., Blayney, L., Livaniou, E., Nounesis, G., Lai, F. A. and Nomikos, M. (2015) Distinctive malfunctions of calmodulin mutations associated with heart RyR2-mediated arrhythmic disease. Biochim. Biophys. Acta 1850, 2168-2176

26 Nomikos, M., Blayney, L. M., Larman, M. G., Campbell, K., Rossbach, A., Saunders, C. M., Swann, K. and Lai, F. A. (2005) Role of phospholipase C- $\zeta$ domains in Ca2+-dependent phosphatidylinositol 4, 5bisphosphate hydrolysis and cytoplasmic Ca2+oscillations. J. Biol. Chem. 280, 31011-31018

27 Fersht, A. (1999) Structure and mechanism in protein science : a guide to enzyme catalysis and protein folding. W.H. Freeman, New York

28 Shaffer, J. F., Kensler, R. W. and Harris, S. P. (2009) The myosin-binding protein C motif binds to F-actin in a phosphorylation-sensitive manner. J. Biol. Chem. 284, 12318-12327

29 Poon, K. L. and Brand, T. (2013) The zebrafish model system in cardiovascular research: A tiny fish with mighty prospects. Glob. Cardiol. Sci. Pract. 4, 9-28

30 Olivotto, I., Maron, M. S., Autore, C., Lesser, J. R., Rega, L., Casolo, G., De Santis, M., Quarta, G., Nistri, S. and Cecchi, F. (2008) Assessment and significance of left ventricular mass by cardiovascular magnetic resonance in hypertrophic cardiomyopathy. J. Am. Coll. Cardiol. 52, 559-566

31 Luther, P. K. and Vydyanath, A. (2011) Myosin binding protein-C: an essential protein in skeletal and cardiac muscle. J. Muscle Res. Cell Motil. 31, 303-305

32 Krishnamoorthy, N., Gajendrarao, P., Olivotto, I. and Yacoub, M. (2017) Impact of diseasecausing mutations on inter-domain interactions in cMyBP-C: a steered molecular dynamics study. J. Biomol. Struct. Dyn. 35, 1916-1922

33 Brown, L. J., Singh, L., Sale, K. L., Yu, B., Trent, R., Fajer, P. G. and Hambly, B. D. (2002) Functional and spectroscopic studies of a familial hypertrophic cardiomyopathy mutation in Motif $\mathrm{X}$ of cardiac myosin binding protein-C. Eur. Biophys. J. 31, 400-408

34 Michalek, A. J., Howarth, J. W., Gulick, J., Previs, M. J., Robbins, J., Rosevear, P. R. and Warshaw, D. M. (2013) Phosphorylation modulates the mechanical stability of the cardiac myosin-binding protein C motif. Biophys. J. 104, 442-452

35 Schlossarek, S., Mearini, G. and Carrier, L. (2011) Cardiac myosin-binding protein C in hypertrophic cardiomyopathy: mechanisms and therapeutic opportunities. J. Mol. Cell. Cardiol. 50, 613620

36 Rafael, J. F., Cruz Filho, F. E. d. S., Carvalho, A. C. C. d., Gottlieb, I., Cazelli, J. G., Siciliano, A. P. and Dias, G. M. (2017) Myosin-binding Protein C Compound Heterozygous Variant Effect on the Phenotypic Expression of Hypertrophic Cardiomyopathy. Arq. Bras. Cardiol. 108, 354-360

37 Lopes, L. R., Rahman, M. S. and Elliott, P. M. (2013) A systematic review and meta-analysis of genotype-phenotype associations in patients with hypertrophic cardiomyopathy caused by sarcomeric protein mutations. Heart 99, 1800-1811 
38 Teekakirikul, P., Kelly, M. A., Rehm, H. L., Lakdawala, N. K. and Funke, B. H. Inherited Cardiomyopathies. J. Mol. Diagn. 15, 158-170

39 Hodatsu, A., Konno, T., Hayashi, K., Funada, A., Fujita, T., Nagata, Y., Fujino, N., Kawashiri, M.-a. and Yamagishi, M. (2014) Compound heterozygosity deteriorates phenotypes of hypertrophic cardiomyopathy with founder MYBPC3 mutation: evidence from patients and zebrafish models. J. Physiol. Heart Circ. Physiol. 307, 1594-1604

40 Lopes, L. R., Zekavati, A., Syrris, P., Hubank, M., Giambartolomei, C., Dalageorgou, C., Jenkins, S., McKenna, W., Plagnol, V. and Elliott, P. M. (2013) Genetic complexity in hypertrophic cardiomyopathy revealed by high-throughput sequencing. J. Med. Genet. 50, 228-239

41 Previs, M. J., Prosser, B. L., Mun, J. Y., Previs, S. B., Gulick, J., Lee, K., Robbins, J., Craig, R., Lederer, W. and Warshaw, D. M. (2015) Myosin-binding protein C corrects an intrinsic inhomogeneity in cardiac excitation-contraction coupling. Sci. Adv. 1, e1400205

42 Da'as, S. I., Yu, J., Butcher, J. T., Krishnamoorthy, N., Al Suwaidi, J. A. S., Kassem, H., Al Shafai, K. N., Al-Hashemi, M. A., Shuayb, L. and Brand, T. (2014) Different human mutations in the myosin binding protein C3 (MYBPC3) produce specific cardiac phenotypes in the zebrafish. Circulation 130, 17545

43 Schlossarek, S. and Carrier, L. (2011) The ubiquitin-proteasome system in cardiomyopathies. Curr. Opin. Cardiol. 26, 190-195

44 Pfuhl, M. and Gautel, M. (2012) Structure, interactions and function of the N-terminus of cardiac myosin binding protein C (MyBP-C): who does what, with what, and to whom? J. Muscle Res. Cell Motil. 33, 83-94

45 Kensler, R. W., Shaffer, J. F. and Harris, S. P. (2011) Binding of the N-terminal fragment C0-C2 of cardiac MyBP-C to cardiac F-actin. J. Struct. Biol. 174, 44-51

46 Belknap, B., Harris, S. P. and White, H. D. (2014) Modulation of thin filament activation of myosin ATP hydrolysis by N-terminal domains of cardiac myosin binding protein-C. Biochemistry 53, 6717-6724

47 Bhuiyan, M. S., Gulick, J., Osinska, H., Gupta, M. and Robbins, J. (2012) Determination of the critical residues responsible for cardiac myosin binding protein C's interactions. J. Mol. Cell. Cardiol. 53, 838-847

48 De Lange, W. J., Grimes, A. C., Hegge, L. F., Spring, A. M., Brost, T. M. and Ralphe, J. C. (2013) E258K HCM-causing mutation in cardiac MyBP-C reduces contractile force and accelerates twitch kinetics by disrupting the cMyBP-C and myosin S2 interaction. J. Gen. Physiol. 142, 241-255

\section{Table Legends}

Table 1. Hypertrophic cardiomyopathy associated c-MYBPC3 missense mutations location, classification and clinical characteristics. a: variant (V) reported at ClinVar database (https://www.ncbi.nlm.nih.gov/clinvar/), b: V5, patient carrying double mutation of V3 and V4, c: reference sequence NM_000256.3, d: protein sequence NP_000247.2, e: frequency allele reported at ExAC $=$ Exome Aggregation Consortium (http://exac.broadinstitute.org), N/R (not reported), f: age of disease onset, g: cardiac septum thickness, h: interpretation of sequence 
variants pathogenicity based on American College of Medical Genetics and Genomics (ACMG) guidance.

Table 2. Secondary structure elements for all recombinant proteins at $25^{\circ} \mathrm{C}$.

Table 3. Thermodynamic parameters calculated from chemical denaturation experiments for WT and MyBP-C variants.

\section{Figure Legends}

Figure 1. Location of hypertrophic cardiomyopathy associated mutations. Schematic illustration showing A. Cardiac myosin binding protein $\mathrm{C} 3$ ( $c-M Y B P C 3)$, showing the location of hypertrophic cardiomyopathy variants in Human Genetics Variants Society (HGVS) format, The c-MYBPC3 is composed of 35 exons, where these variants are located within exon 5, 6 and 13. B. MyBP-C protein consists of 11 domains $(\mathrm{C} 0-\mathrm{C} 10) ; 8$ immunoglobulin-like domains (IgC2-like) and 3 fibronectin type III-like (FN3) domains with N-terminal Proline-Alanine rich linker sequence and the regulatory Motif that includes the phosphorylation sites indicated by asterisks. All variants in this study are within the C1-C2 domains.

Figure 2. Expression of $c-M Y B P C 3$ variants in the zebrafish model recapitulated the human HCM phenotype. A. Zebrafish injected with human $c-M Y B P C 3$ synthetic RNA of wild type and the five variants at one cell stage. At 72 hours post fertilization, larvae were mounted posteriorly to image ventricle chamber of the heart (black box). B. Representative images of aberrant heart morphology of the five expressed variants in comparison to control group. C. Cardiac functional analysis of variants expressed in zebrafish, the measurement of ventricular diastolic myocardial thickness for V5 (double mutation E441K \& E258K) and V3 (E258K) were the most significantly increased mean followed by V1 (R177H), V4 (E441K) and V2 (A216T) in comparison to WT. D. Ventricular systolic myocardial thickness measurement for V5 were the most significantly increased mean at $14.6 \mu \mathrm{m}$ followed by V3, V1, V4 and V2 in comparison to WT. E. Heart contractile measurement showed that both V5 and V3 significantly impacted the 
average heart rate in injected zebrafish demonstrated by the significant reduced heart rate mean measured by beats per minute (bpm) compared to WT. While, V1, V2 and V4 heart rates were still less than the control zebrafish. Cardiac function analyses were represented in Box - Whisker plots and analysis using one-way ANOVA multiple comparisons test. Values were expressed as means $\pm \mathrm{SE}$ and $p$ values of $<0.05$ were considered statistically significant, a value of $* * \mathrm{p}<0.01$, $* * * \mathrm{p}<0.001$ and $* * * \mathrm{p}<0.0001$.

Figure 3. Molecular model of the structural analysis of V5 and V3. A. MyBP-C WT 3D structure. B. Structural comparison of WT vs V3. C. Structural comparison of WT vs V5. Both V5 and V3 deviated structurally and rearranged differently from the WT illustrated mainly in elements rearrangement at the motif region. D. Root mean square deviation (RMSD) calculation of the representative structures from molecular dynamics simulations illustrated that V3 conformational deviation appeared to be flexible, while V5 showed more rigid behavior. E. Secondary structural change analysis of intra-molecular consequences. V3 model illustrated reduced $\beta$-sheets content and increased coils content compared to WT, however, V5 showed inverse behavior. This analysis correlated with the deviation analysis and explained the flexibility of V3 as there is increase of coils and for the rigidity of V5 due to the reduction of coils and increase of $\beta$-sheets contents.

Figure 4. Expression, purification and intein cleavage of $\mathrm{C} 0-\mathrm{C} 2$ (MyBP-C) recombinant protein fragment. A. Schematic diagram showing the $\mathrm{C} 0-\mathrm{C} 2$ domains fragment and its proposed interaction with cardiac actin. B. Analysis of recombinant expression and purification of $\mathrm{C} 0-\mathrm{C} 2$ fragment protein of WT, V1, V2, V3, V4 and V5 (V3+V4) of equivalent volumes at $12 \%$ SDS-PAGE by Coomassie staining and western blot with a polyclonal MyBP-C antibody (1:1000 dilution). C. MyBP-C circular dichroism (CD) spectra measurements at $25^{\circ} \mathrm{C}$. All variants disrupted the structure of the protein fragment leading to conformations with altered secondary structure characteristics impacting the fragment elements; predominantly the $\beta$-sheet, random coil content and helical content when compared to WT. However, V5 (double mutation) resulted in the most altered secondary structure in comparison to WT. (WT:black, V1:red, V2:green, V3:blue, V4:cyan and V5:pink). 
Figure 5. Thermal denaturation profile of MyBP-C recombinant protein fragments measured as normalized CD signal at $208 \mathbf{~ n m}$. The thermal denaturation curve of WT, V1, V2, V3 and V4 reveals the presence of two consecutive unfolding transitions at which the first thermal transition occurs in the temperature range $50^{\circ} \mathrm{C}-65^{\circ} \mathrm{C}$ and the second in the temperature range $65^{\circ} \mathrm{C}-90^{\circ} \mathrm{C}$. while, in contrast, the melting profile of V5 indicated only the first unfolding transition at $50^{\circ} \mathrm{C}-65^{\circ} \mathrm{C}$, indicating that no further aggregation occurs after $\sim 60^{\circ} \mathrm{C}$ for this fragment.

Figure 6. Chemical denaturation trace of MyBP-C recombinant protein fragments. Changes in the weighted average emission wavelength (excitation $295 \mathrm{~nm}$ ) of MyBP-C WT and the different variants were measured in solution upon titration with increasing amounts of the chemical denaturant guanidine hydrochloride $(\mathrm{GuHCl})$. All MyBP-C fragments had displayed reversible transition presented by Sigmoidal two-state denaturation curves for the fraction of unfolding. Calculated Gibbs free energy change $(\Delta G)$ of the unfolding process illustrated increased stability for all variants except V5 in comparison to WT.

Figure 7. Co-sedimentation binding assays of $\mathrm{MyBP}-\mathrm{C} \mathrm{CO}-\mathrm{C} 2$ recombinant proteins and cardiac actin (c-actin). MyBP-C C0-C2 recombinant proteins $(2 \mu \mathrm{M})$ were incubated with cactin at RT for $30 \mathrm{~min}$ and spun for 1 hour at 14,000 g. The amount of MyBP-C C0-C2 proteins in supernatant (unbound fraction) was analyzed by SDS-PAGE and quantified by Coomassie brilliant blue and densitometric analysis. (A) Optimization experiments using $2 \mu \mathrm{M}$ of MyBP-C $\mathrm{C} 0-\mathrm{C} 2 \mathrm{WT}$ and 20 or $30 \mu \mathrm{M}$ c-actin showing that saturated binding is achieved with $30 \mu \mathrm{M} \mathrm{c}-$ actin. (B) Representative SDS-PAGE gel and Coomassie brilliant blue staining following MyBP$\mathrm{C}$ C0-C2 mutant protein-c-actin co-sedimentation assays showing the amounts of MyBP-C C0$\mathrm{C} 2$ mutant proteins in supernatant fractions (unbound fraction) pre (-) and post (+) cosedimentation with $30 \mu \mathrm{M}$ c-actin. (C) Densitometric analysis and \% normalization of the binding of MyBP-C C0-C2 recombinant proteins to $30 \mu \mathrm{M}$ c-actin. Significant effects (asterisks) were calculated by comparison to the binding of WT MyBP-C C0-C2 using an unpaired Student's t-test. $\mathrm{n}=2 \pm$ S.E.M., ${ }^{*} \mathrm{p}<0.05$ and ${ }^{*} \mathrm{p}<0.01,(\mathrm{GraphPad}$ Prism 7$)$. 


\section{Tables}

\section{Table 1}

\begin{tabular}{|c|c|c|c|c|c|c|c|c|c|}
\hline $\begin{array}{l}\text { Variant }^{\mathrm{a}} \\
\text { (V) }\end{array}$ & $\mathrm{c}-\mathrm{MYBPC}^{\mathrm{c}}$ & MyBP-C $^{d}$ & $\begin{array}{l}\text { ExAC allele } \\
\text { frequency }\end{array}$ & MyBP-C Domain & Years $^{f}$ & $\begin{array}{c}\text { Thickness }^{\mathrm{g}} \\
\text { (mm) }\end{array}$ & $\begin{array}{l}\text { HCM patients } \\
\text { clinical symptoms }\end{array}$ & $\begin{array}{l}\text { Classification of } \\
\text { Pathogenicity }\end{array}$ & Reference \\
\hline V1 & c. $530 \mathrm{G}>\mathrm{A}$ & p.Arg177His & 0.0009493 & $\begin{array}{l}\text { C1 (Ig-like C2-type } \\
\text { 1) }\end{array}$ & 56 & 15 & Chest pain & $\begin{array}{l}\text { Unlikely to be } \\
\text { pathogenic }\end{array}$ & $\begin{array}{l}\text { (Jaafar, Gómez } \\
\text { et al. 2016) }\end{array}$ \\
\hline $\mathrm{V} 2$ & c. $646 \mathrm{G}>\mathrm{A}$ & p.Ala216Thr & 0.000365 & $\begin{array}{l}\text { C1 (Ig-like C2-type } \\
\text { 1) }\end{array}$ & 34 & $\mathrm{~N} / \mathrm{R}$ & $\begin{array}{l}\text { Sub-aortic } \\
\text { occlusion }\end{array}$ & $\begin{array}{l}\text { Class 3- } \\
\text { Unknown } \\
\text { pathogenicity }\end{array}$ & $\begin{array}{l}\text { (Rodríguez- } \\
\text { García, } \\
\text { Monserrat et al. } \\
\text { 2010) }\end{array}$ \\
\hline V3 & c. $772 \mathrm{G}>\mathrm{A}$ & p.Glu258Lys & $3.9 \mathrm{E}-05$ & $\begin{array}{l}\text { M-motif } \\
\text { (phosphorylation site) }\end{array}$ & 49 & 15 & $\begin{array}{l}\text { chest pain and } \\
\text { palpitation, chest } \\
\text { pain and dyspnea }\end{array}$ & $\begin{array}{l}\text { Class 5- } \\
\text { Certainly } \\
\text { pathogenic }\end{array}$ & $\begin{array}{l}\text { (Jaafar, Gómez } \\
\text { et al. 2016) }\end{array}$ \\
\hline V4 & c. $1321 \mathrm{G}>\mathrm{A}$ & p.Glu441Lys & 0.000162 & $\begin{array}{l}\text { C2 (Ig-like C2-type } \\
\text { 2) }\end{array}$ & 45 & 23.2 & $\begin{array}{l}\text { no syncope, } \\
\text { complaint about } \\
\text { angina at exertion, } \\
\text { but no ventricular } \\
\text { tachycardia }\end{array}$ & $\begin{array}{l}\text { Class 3- } \\
\text { Unknown } \\
\text { pathogenicity }\end{array}$ & $\begin{array}{l}\text { (Marsiglia, } \\
\text { Batitucci et al. } \\
\text { 2010) }\end{array}$ \\
\hline $\begin{array}{l}\mathrm{V}^{\mathrm{b}} \\
\left(\mathrm{V} 3^{+}\right. \\
\mathrm{V} 4)^{-}\end{array}$ & $\begin{array}{l}\text { c. } 772 \mathrm{G}>\mathrm{A} \\
+ \text { c. } 1321 \mathrm{G}>\mathrm{A}\end{array}$ & $\begin{array}{l}\text { p.(Glu258Lys } \\
+ \text { Glu441Lys) }\end{array}$ & - & $\begin{array}{l}\text { M motif and C2 } \\
\text { (Ig-like C2-type 2) }\end{array}$ & 21 & 22 & $\begin{array}{l}\text { dyspnea, } \\
\text { progressive heart } \\
\text { failure with } \\
\text { repeated } \\
\text { hospitalizations } \\
\text { then patient died at } \\
\text { age } 42\end{array}$ & $\begin{array}{l}\text { Class 5- } \\
\text { Certainly } \\
\text { pathogenic }\end{array}$ & $\begin{array}{l}\text { (Gajendrarao, } \\
\text { Krishnamoorthy } \\
\text { et al. 2015) }\end{array}$ \\
\hline
\end{tabular}


Table 2

\begin{tabular}{|c|c|c|c|c|c|}
\hline \multirow{2}{*}{ Protein } & \multirow{2}{*}{$\alpha$-Helix } & \multicolumn{2}{|c|}{$\beta$-Sheet } & \multirow{2}{*}{$\beta$-Turn } & \multirow{2}{*}{ Random Coil } \\
\cline { 3 - 6 } & & Antiparallel & Parallel & & \\
\hline WT & $20.7 \%$ & $11.3 \%$ & $13.6 \%$ & $18.9 \%$ & $40.2 \%$ \\
\hline V1 & $26.8 \%$ & $21.2 \%$ & $8.9 \%$ & $19.1 \%$ & $28.1 \%$ \\
\hline V2 & $26.4 \%$ & $11.1 \%$ & $10.6 \%$ & $18.4 \%$ & $36.4 \%$ \\
\hline V3 & $26.8 \%$ & $14.4 \%$ & $9.9 \%$ & $18.2 \%$ & $33.7 \%$ \\
\hline V4 & $26.0 \%$ & $10.6 \%$ & $10.7 \%$ & $18.4 \%$ & $38.5 \%$ \\
\hline V5 & $26.8 \%$ & $21.2 \%$ & $8.9 \%$ & $19.1 \%$ & $28.5 \%$ \\
\hline
\end{tabular}

Table 3

\begin{tabular}{|c|c|c|c|}
\hline \multirow{2}{*}{ Protein } & $\mathrm{m}$ & $\mathrm{D}^{50 \%}$ & $\Delta \mathrm{G}_{\mathrm{DN}}$ \\
& $\left(\mathrm{kcal} \mathrm{mol}^{-1} \mathrm{M}^{-1}\right)$ & $(\mathrm{M})$ & $3.46 \pm 0.27$ \\
\hline WT & $2.07 \pm 0.15$ & $1.67 \pm 0.05$ & $4.07 \pm 0.34$ \\
\hline V1 & $2.45 \pm 0.19$ & $1.66 \pm 0.05$ & $3.98 \pm 0.27$ \\
\hline V2 & $2.25 \pm 0.15$ & $1.77 \pm 0.03$ & $4.90 \pm 0.37$ \\
\hline V3 & $2.90 \pm 0.21$ & $1.69 \pm 0.03$ & $4.98 \pm 0.36$ \\
\hline V4 & $2.83 \pm 0.20$ & $1.76 \pm 0.03$ & $3.17 \pm 0.26$ \\
\hline
\end{tabular}


A

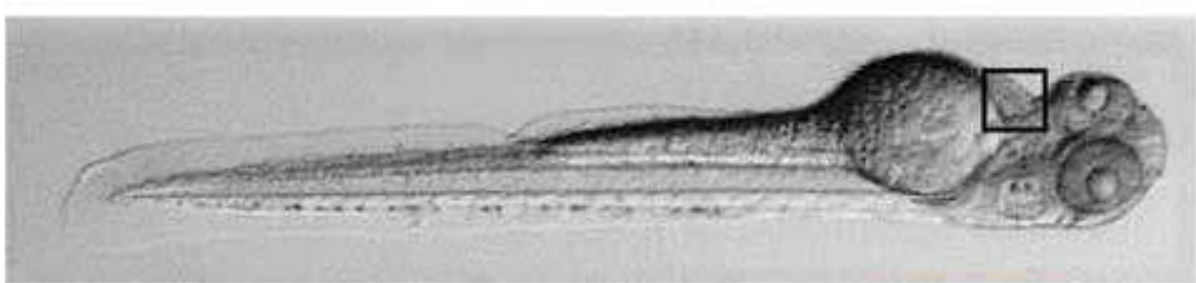

B
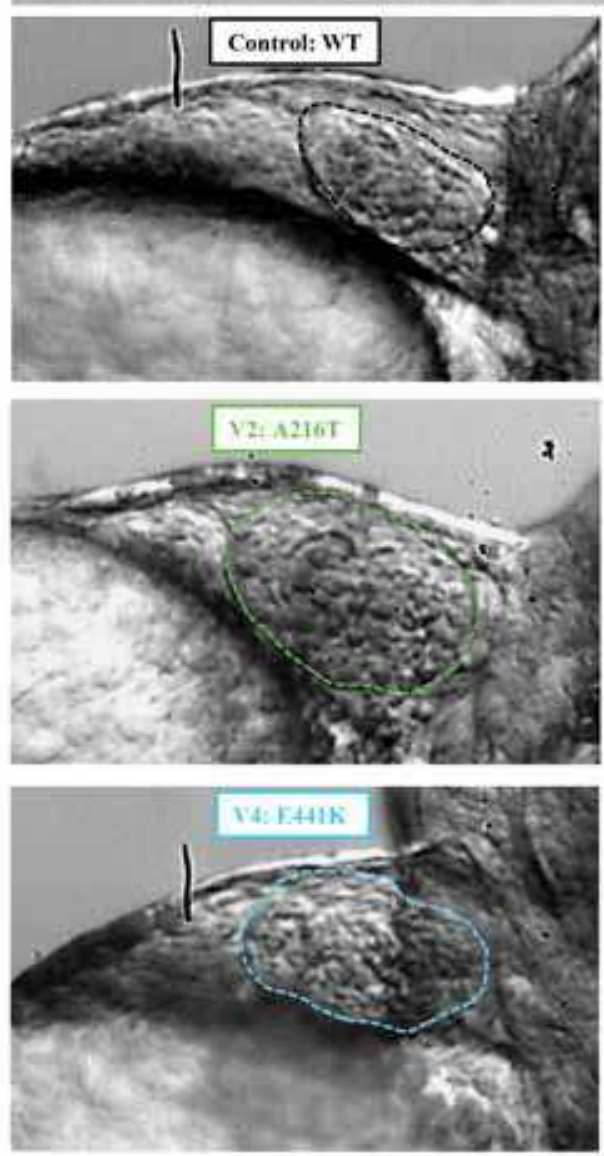
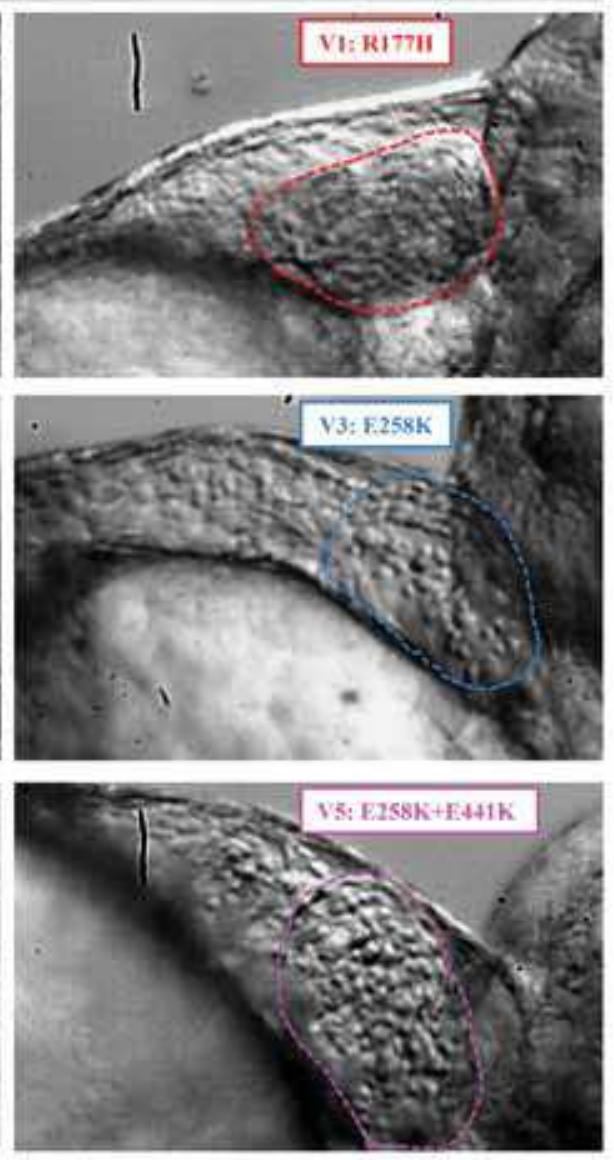

C

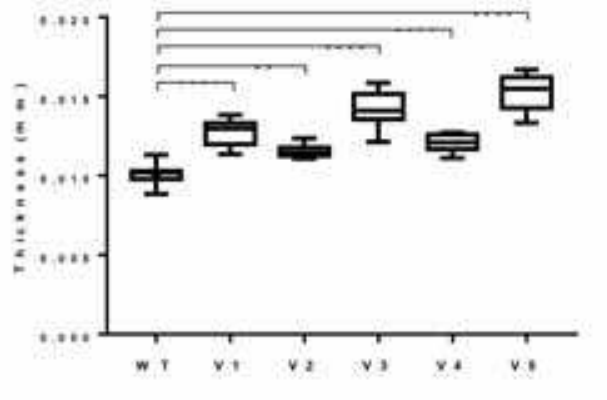

D

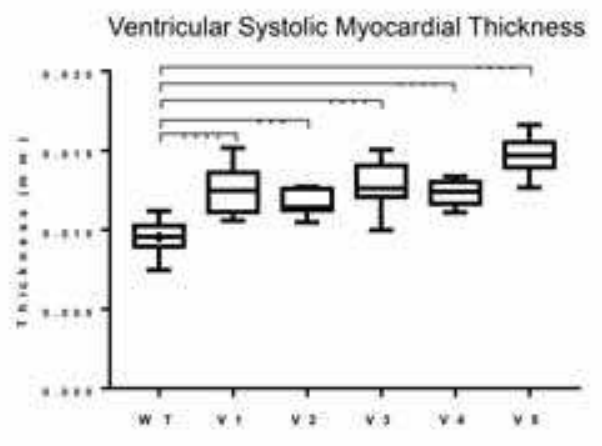

E

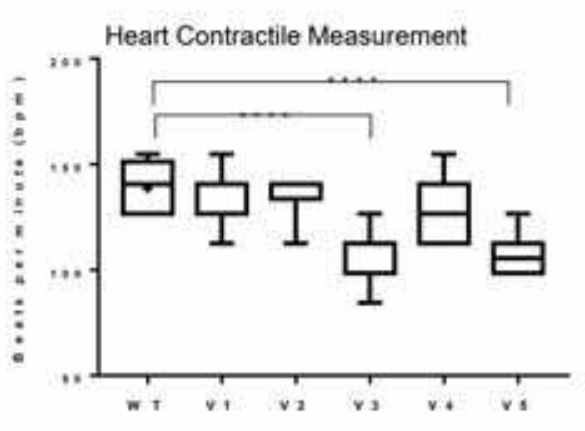



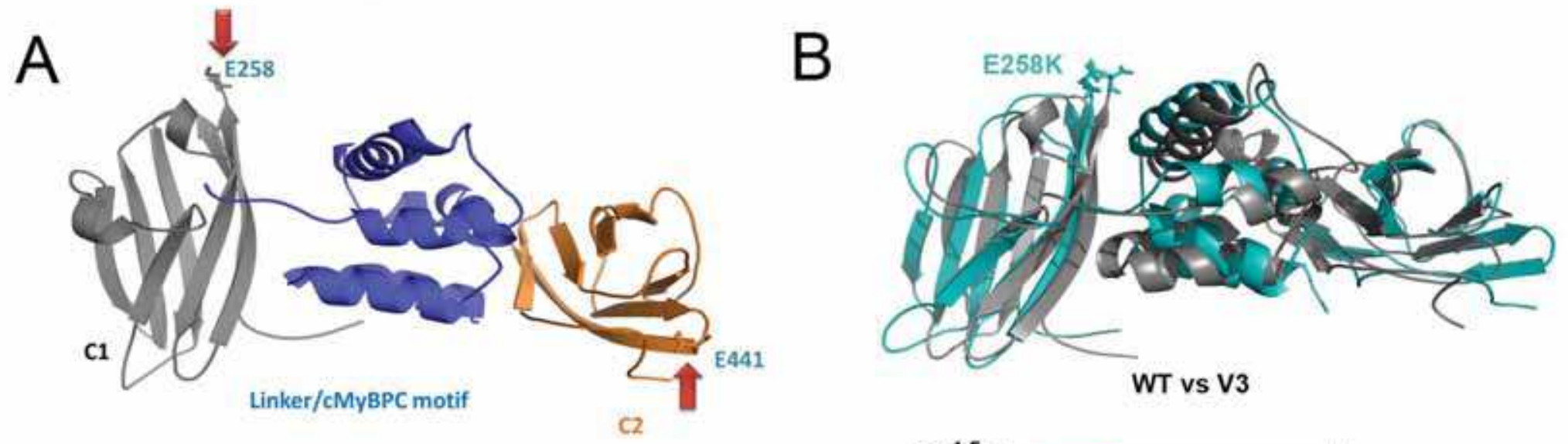

C

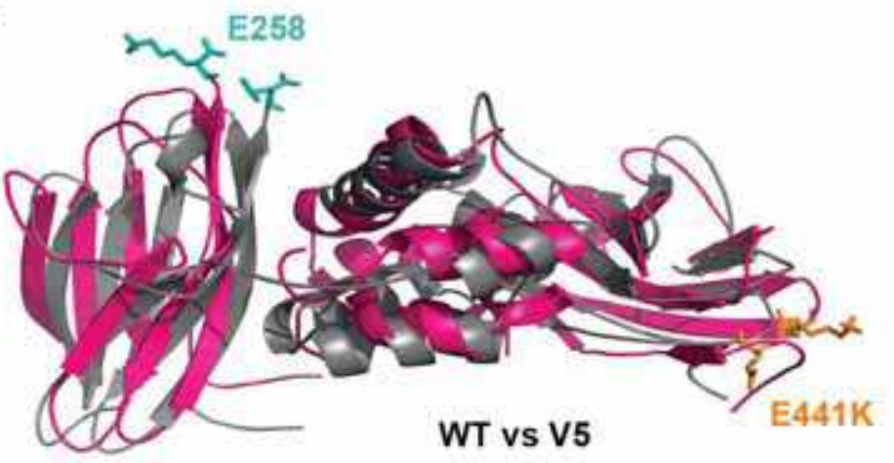

D
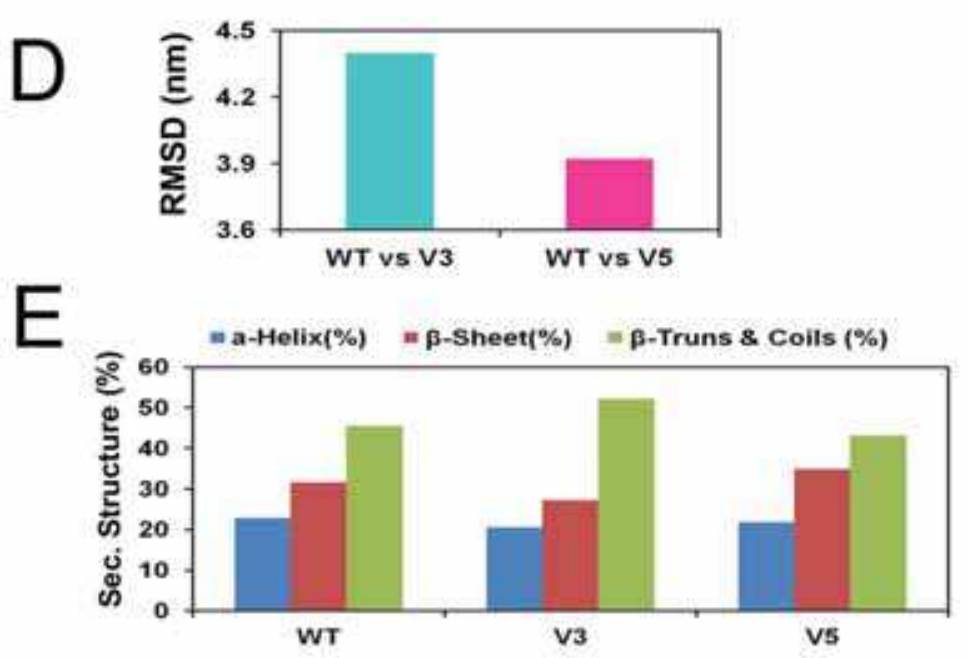
A

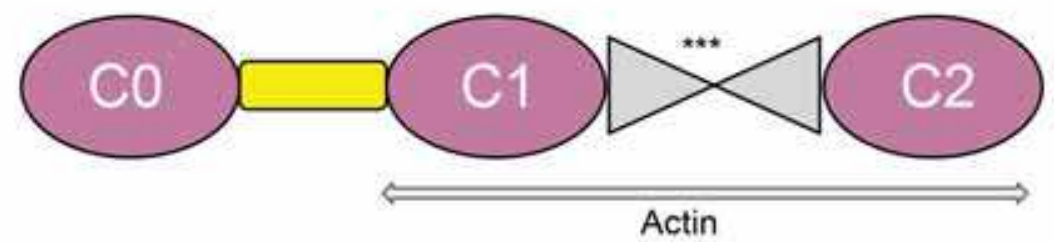

OlgC2-like domain 口Pro-Ala region $\bowtie$ M Motif

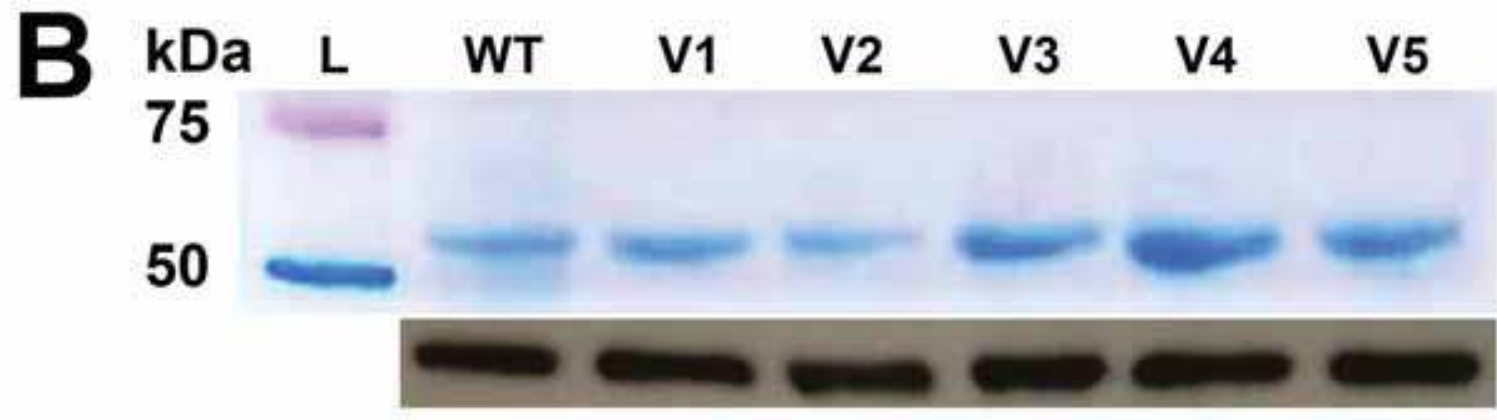

Far CD Spectra $\left(25^{\circ} \mathrm{C}\right)$

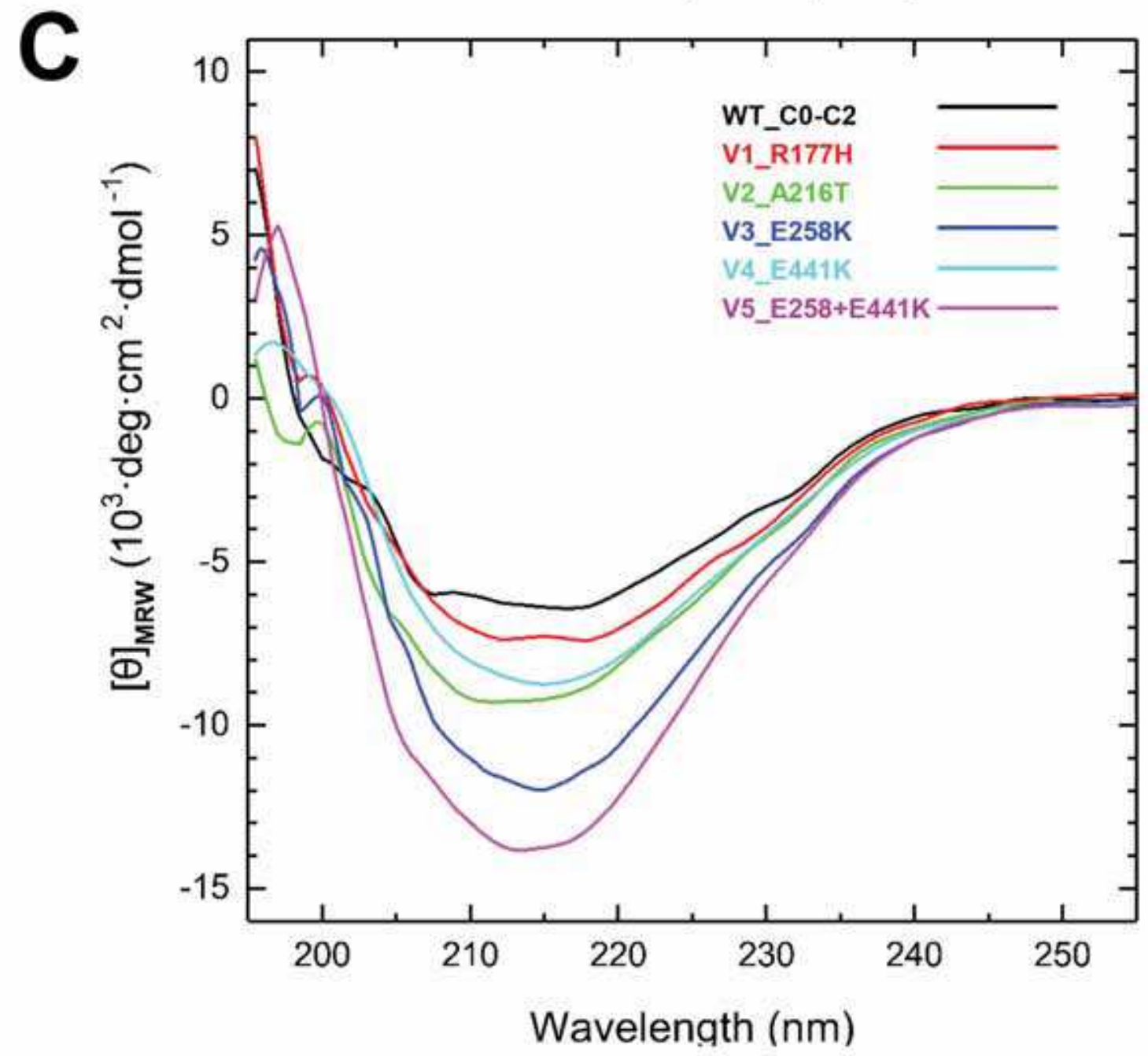



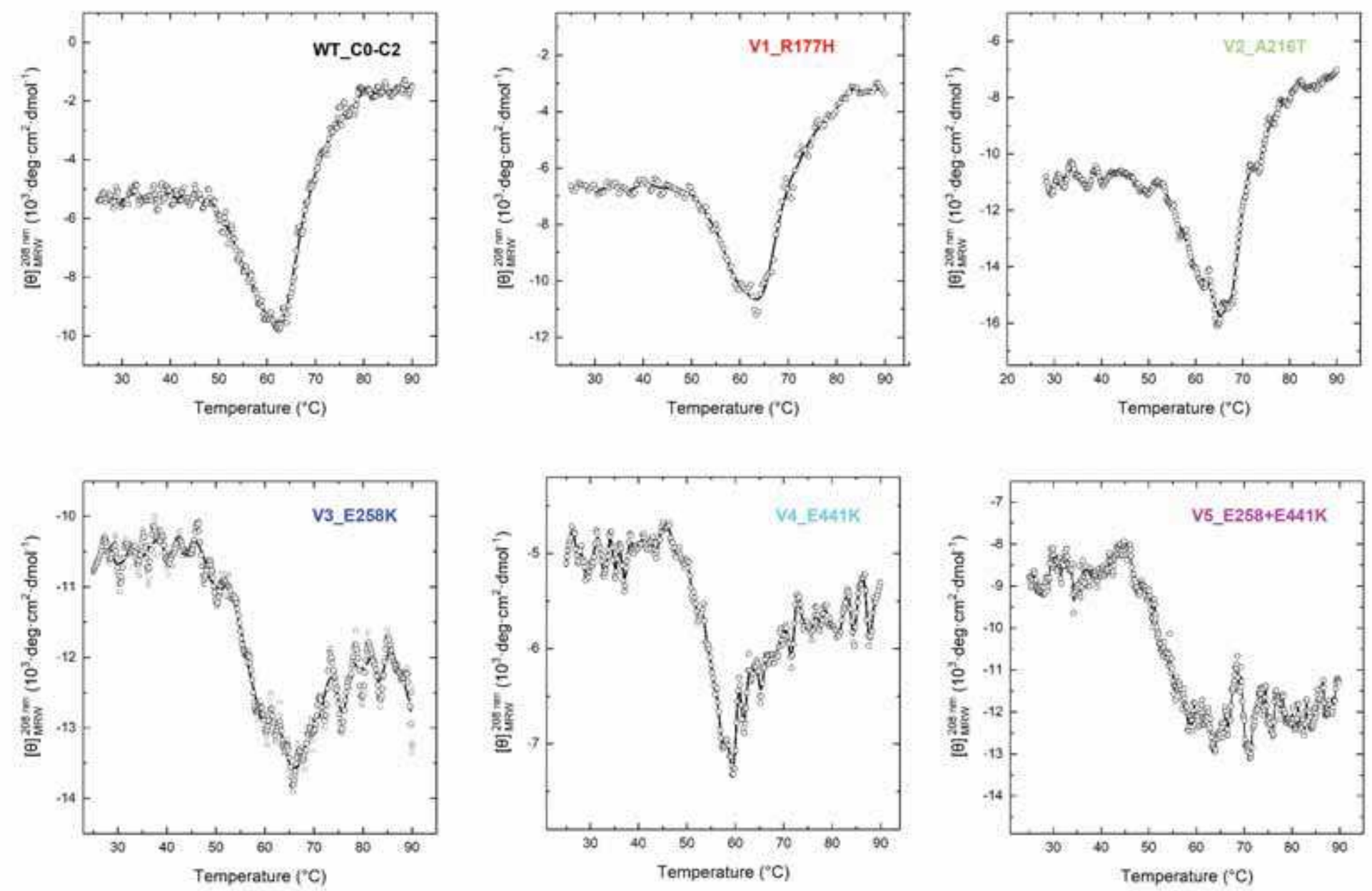

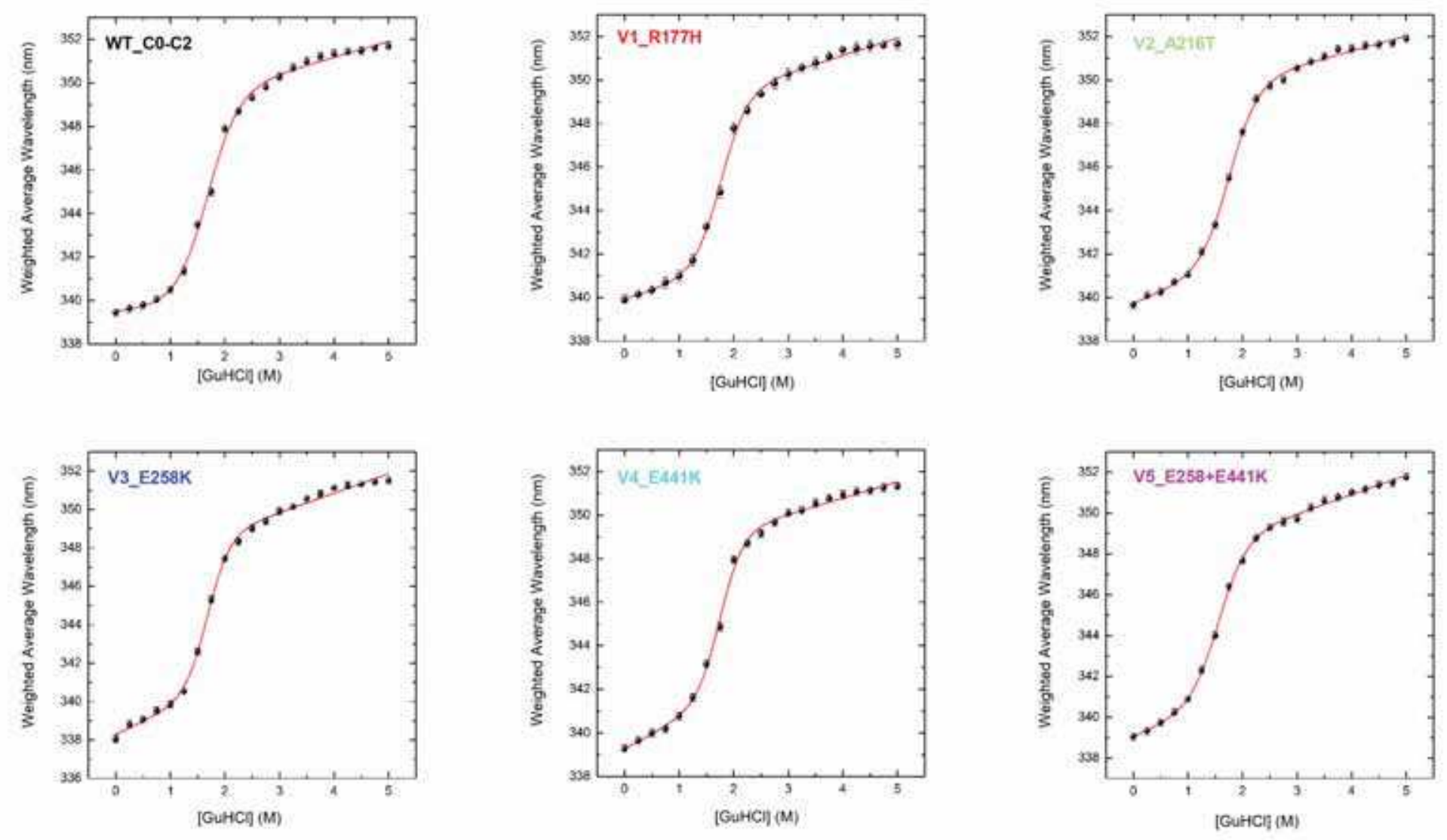
A
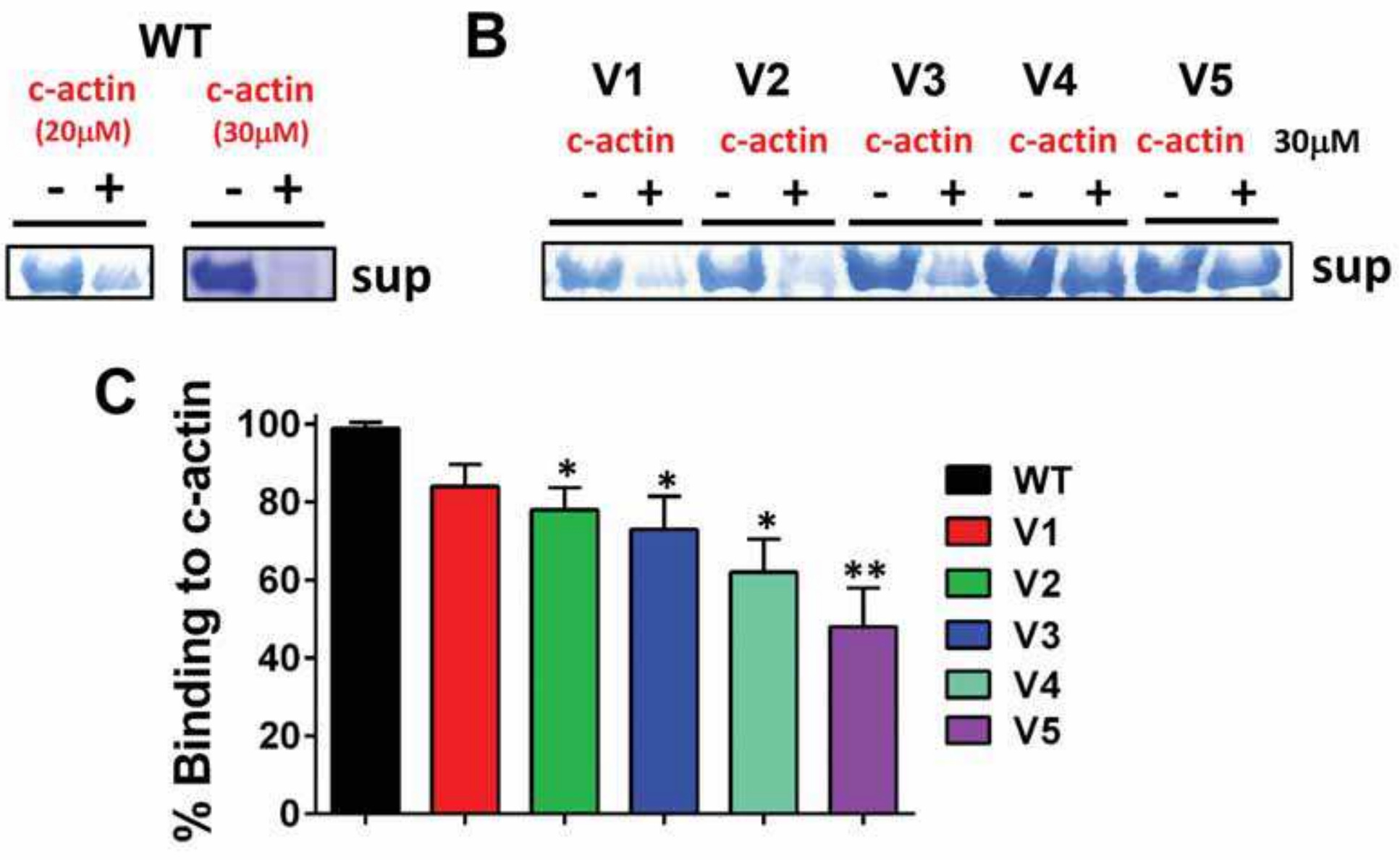


\section{Supplemental Material}

Tables

\begin{tabular}{|c|c|c|c|c|c|}
\hline \multirow{2}{*}{$\begin{array}{c}\text { Temperature } \\
\left({ }^{\circ} \mathrm{C}\right)\end{array}$} & \multirow{2}{*}{$\alpha$-Helix } & \multicolumn{2}{|c|}{$\beta$-Sheet } & \multirow{2}{*}{$\beta$-Turn } & \multirow{2}{*}{ Random Coil } \\
\cline { 3 - 5 } & & Antiparallel & Parallel & & \\
\hline 25 & $20.7 \%$ & $11.3 \%$ & $13.6 \%$ & $18.9 \%$ & $40.2 \%$ \\
\hline 65 & $25.6 \%$ & $9.9 \%$ & $11.7 \%$ & $18.0 \%$ & $38.1 \%$ \\
\hline 90 & $11.1 \%$ & $11.1 \%$ & $12.6 \%$ & $19.4 \%$ & $50.4 \%$ \\
\hline
\end{tabular}

Table 1. Secondary structure elements for WT MyBP-C fragment at various temperatures.

\begin{tabular}{|c|c|c|c|c|c|}
\hline \multirow{2}{*}{$\begin{array}{c}\text { Temperature } \\
\left({ }^{\circ} \mathrm{C}\right)\end{array}$} & \multirow{2}{*}{$\alpha$-Helix } & \multicolumn{2}{|c|}{$\beta$-Sheet } & \multirow{2}{*}{$\beta$-Turn } & \multirow{2}{*}{ Random Coil } \\
\cline { 3 - 5 } & & Antiparallel & Parallel & & \\
\hline 25 & $26.8 \%$ & $21.2 \%$ & $8.9 \%$ & $19.1 \%$ & $28.1 \%$ \\
\hline 65 & $26.2 \%$ & $10.9 \%$ & $10.8 \%$ & $18.2 \%$ & $37.4 \%$ \\
\hline 90 & $12.1 \%$ & $11.6 \%$ & $12.8 \%$ & $19.4 \%$ & $49.1 \%$ \\
\hline
\end{tabular}

Table 2. Secondary structure elements for V1 MyBP-C fragment at various temperatures.

\begin{tabular}{|c|c|c|c|c|c|}
\hline \multirow{2}{*}{$\begin{array}{c}\text { Temperature } \\
\left({ }^{\circ} \mathrm{C}\right)\end{array}$} & \multirow{2}{*}{$\alpha$-Helix } & \multicolumn{2}{|c|}{$\beta$-Sheet } & \multirow{2}{*}{$\beta$-Turn } & \multirow{2}{*}{ Random Coil } \\
\cline { 3 - 5 } & & Antiparallel & Parallel & & \\
\hline 25 & $26.4 \%$ & $11.1 \%$ & $10.6 \%$ & $18.4 \%$ & $36.4 \%$ \\
\hline 65 & $28.7 \%$ & $12.7 \%$ & $9.5 \%$ & $17.8 \%$ & $32.9 \%$ \\
\hline 90 & $17.9 \%$ & $11.2 \%$ & $13.7 \%$ & $19.2 \%$ & $45.3 \%$ \\
\hline
\end{tabular}

Table 3. Secondary structure elements for V2 MyBP-C fragment at various temperatures. 


\begin{tabular}{|c|c|c|c|c|c|}
\hline \multirow{2}{*}{$\begin{array}{c}\text { Temperature } \\
\left({ }^{\circ} \mathrm{C}\right)\end{array}$} & \multirow{2}{*}{$\alpha$-Helix } & \multicolumn{2}{|c|}{$\beta$-Sheet } & \multirow{2}{*}{$\beta$-Turn } & \multirow{2}{*}{ Random Coil } \\
\cline { 3 - 5 } & & Antiparallel & Parallel & & \\
\hline 25 & $26.8 \%$ & $14.4 \%$ & $9.9 \%$ & $18.2 \%$ & $33.7 \%$ \\
\hline 65 & $25.9 \%$ & $17.4 \%$ & $9.4 \%$ & $18.8 \%$ & $29.9 \%$ \\
\hline 90 & $28.6 \%$ & $14.8 \%$ & $9.7 \%$ & $18.2 \%$ & $27.9 \%$ \\
\hline
\end{tabular}

Table 4. Secondary structure elements for V3 MyBP-C fragment at various temperatures.

\begin{tabular}{|c|c|c|c|c|c|}
\hline \multirow{2}{*}{$\begin{array}{c}\text { Temperature } \\
\left({ }^{\circ} \mathrm{C}\right)\end{array}$} & \multirow{2}{*}{$\alpha$-Helix } & \multicolumn{2}{|c|}{$\beta$-Sheet } & \multirow{2}{*}{$\beta$-Turn } & \multirow{2}{*}{ Random Coil } \\
\cline { 3 - 5 } & & Antiparallel & Parallel & & \\
\hline 25 & $26.0 \%$ & $10.6 \%$ & $10.7 \%$ & $18.4 \%$ & $38.5 \%$ \\
\hline 65 & $26.3 \%$ & $11.1 \%$ & $10.6 \%$ & $18.3 \%$ & $36.4 \%$ \\
\hline 90 & $25.7 \%$ & $9.9 \%$ & $11.7 \%$ & $18.1 \%$ & $39.5 \%$ \\
\hline
\end{tabular}

Table 5. Secondary structure elements for V4 MyBP-C fragment at various temperatures.

\begin{tabular}{|c|c|c|c|c|c|}
\hline \multirow{2}{*}{$\begin{array}{c}\text { Temperature } \\
\left({ }^{\circ} \mathrm{C}\right)\end{array}$} & $\alpha$-Helix & \multicolumn{2}{|c|}{$\beta$-Sheet } & \multirow{2}{*}{$\beta$-Turn } & $\begin{array}{c}\text { Random } \\
\text { Coil }\end{array}$ \\
\cline { 3 - 5 } & & Antiparallel & Parallel & & \\
\hline 25 & $26.8 \%$ & $21.2 \%$ & $8.9 \%$ & $19.1 \%$ & $28.5 \%$ \\
\hline 65 & $30.4 \%$ & $20.1 \%$ & $7.6 \%$ & $19.1 \%$ & $26.4 \%$ \\
\hline 90 & $27.9 \%$ & $19.9 \%$ & $8.6 \%$ & $19.6 \%$ & $27.6 \%$ \\
\hline
\end{tabular}

Table 6. Secondary structure elements for V5 MyBP-C fragment at various temperatures. 